\title{
Development of Transportation Infrastructure in the Context of Economic Growth
}

Manuel Benjamin Ortiz-Moctezuma (moctez@iiasa.ac.at), Denis Pivovarchuk (pivovar@iiasa.ac.at), Jana Szolgayova (szolgay@iiasa.ac.at), Sabine Fuss (fuss@iiasa.ac.at)

\section{Approved by}

Arkady Kryazhimskiy (kryazhim@iiasa.ac.at)

Program Leader, Dynamic Systems

November 2008 


\begin{abstract}
Developed road infrastructure is an essential factor facilitating and accelerating economic growth, which will in turn enable the addition of more roads. At the same time, the marginal benefit of adding roads to a large stock of existing capacity might be diminishing. It is thus evident that the co-evolution of economic output and road infrastructure is rather intricate and deserves special attention. The model developed in this paper therefore investigates the interdependency between a country's economic growth and the development of transportation infrastructure in this country. To this end, a co-evolutionary perspective is developed, where the mutual influence of the rate of economic growth and the capacity of transportation infrastructure are explicitly taken into account. This approach enables us to set up an optimal control problem, where the optimal investment rate is determined considering the co-evolutionary dynamics of GDP growth and capacity expansion. This model forms a comprehensive framework for understanding the underlying dynamics and the patterns of economic growth in relation to transport infrastructure. We find an analytical solution for the infinite horizon problem, where the control turns out to be a constant. The steady state is shown to depend crucially on the rate of physical decay of roads, which we think can be interpreted as an index of quality, and the speed of adjustment, at which the economy moves along a trajectory. Testing the model for the data of two countries, France and Finland, illustrates the usefulness of such an approach to real world problems and possibly policy recommendation, where the model would have to be adapted to the peculiarities of each country or region to make precise statements.
\end{abstract}

Key words: road infrastructure, investment, economic growth, optimum control theory, co-evolutionary modeling

JEL Classification: C 61, H54, O1 


\author{
About the Authors \\ Manuel Benjamin Ortiz-Moctezuma \\ Colosio Fellow \\ Dynamic Systems Program \\ International Institute of Systems Analysis \\ Schlossplatz 1, 2361 Laxenburg, Austria \\ and \\ Universidad Politecnica de Victoria, Mexico
}

\author{
Denis Pivovarchuk \\ Faculty of Computation Mathematics and Cybernetics \\ Moscow State University, Russia
}

Jana Szolgayova

Forestry Program

International Institute of Systems Analysis

Schlossplatz 1, 2361 Laxenburg, Austria

and

Department of Applied Mathematics and Statistics

Faculty of Mathematics, Physics and Informatics

Comenius University, Bratislava, Slovakia

Sabine Fuss

Forestry Program

International Institute of Systems Analysis

Schlossplatz 1, 2361 Laxenburg, Austria

\title{
Acknowledgments
}

This work was carried out as an intersection of the Driving Forces of Economic Growth (ECG) Project and the Fragility of Critical Infrastructures (FCI) initiative at IIASA. The authors are grateful for the attention, suggestions and useful comments from all researchers of the Dynamic Systems Program (DYN), Michael Obersteiner (FOR), Jerry Silverberg (TNT), and most of all Arkady Kryazhimskiy (DYN), who initiated this study. 


\section{Contents}

1 Introduction $\quad 1$

2 Optimal Control Approach to Infrastructure Investment \& Economic Growth

2.1 Model . . . . . . . . . . . . . . . . . . . . . . . . 4

2.2 Specifying Functions . . . . . . . . . . . . . . . . 6

2.3 The Share of Road Infrastructure in Economic Output . . . . . . . . . . 8

2.4 Solution of Optimal Control Problem . . . . . . . . . . . . . . . 9

3 Data \& Calibration $\quad 14$

3.1 Calibration Methods . . . . . . . . . . . . . . . . . . 14

3.2 Calibration Results . . . . . . . . . . . . . . . . . . . 16

4 Optimal Control Results: Country Case Studies $\quad 18$

4.1 Infrastructure Quality \& Steady State GDP . . . . . . . . . . . . . . . 19

4.2 The Speed of Adjustment \& Steady State GDP . . . . . . . . . . . . . . . 22

4.3 The Share of Road Infrastructure in GDP \& Steady State GDP . . . . . . . 23

5 Summary and Conclusion $\quad 25$

$\begin{array}{ll}\text { References } & 26\end{array}$

A Appendix: Optimal Control Problem with Finite Time Horizon $\quad 27$

B Appendix: Some Additional Data 28 


\title{
Development of Transportation Infrastructure in the Context of Economic Growth
}

\author{
Manuel Benjamin Ortiz-Moctezuma (moctez@iiasa.ac.at), Denis \\ Pivovarchuk(pivovar@iiasa.ac.at), Jana Szolgayova \\ (szolgay@iiasa.ac.at), Sabine Fuss (fuss@iiasa.ac.at)
}

\section{Introduction}

Developed road infrastructure is an essential factor facilitating and accelerating economic growth, which will in turn enable the addition of more roads. At the same time, the marginal benefit of adding roads to a large stock of existing capacity might be diminishing. It is thus evident that the co-evolution of economic output and road infrastructure is rather intricate and deserves special attention. The model developed in this paper therefore investigates the interdependency between a country's economic growth and the development of transportation infrastructure in this country. To this end, a co-evolutionary perspective is developed, where the mutual influence of the rate of economic growth and the capacity of transportation infrastructure are explicitly taken into account. This approach enables us to set up an optimal control problem, where the optimal investment rate is determined considering the co-evolutionary dynamics of GDP growth and capacity expansion. This model forms a comprehensive framework for understanding the underlying dynamics and the patterns of economic growth in relation to transport infrastructure.

Following the seminal work by Aschauer $(1989)^{1}$, interest in the relationship between economic growth and infrastructure had been rekindled and, as a consequence, a large body of mainly empirical studies emerged from the effort of the research community also in response to demand for better insights from the side of policy-makers. Gramlich (1994) and later Button (1998) provide in-depth reviews of this work and also some valid criticism with respect to both methodology and - more importantly - the underlying mechanism of the dynamics. ${ }^{2}$ One debate in the latter context is, for example, the question of causality involved in these processes, i.e. whether economic growth is accelerated by increases in the stock of infrastructure or whether additions to existing infrastructure are caused by enhanced economic growth. Methodologically, a point of criticism is that crosscountry analysis is barely even possible given the differences in measurement practices and infrastructure definitions in official accounting data. To this add the differences in maintenance and utilization of infrastructure. More specifically, Gramlich (1994) claims that a sectorial view has to be taken, rather than an aggregate perspective, which would only give blurred results, as different types of infrastructure affect growth to varying extents and in different ways. Finally, it is not even clear what the best approach to such empirical estimates is: if it is even admissible to employ a production function as many authors do,

\footnotetext{
${ }^{1}$ Aschauer's (1989) research starts from the conviction that public investment of a specific type could have a larger effect on productivity than others and finds that some types of infrastructure ("core" infrastructure) indeed have very high marginal productivity.

${ }^{2}$ Gramlich (1994) discusses most lines of criticism and Button (1998) summarizes and extends this list.
} 
for instance. Compared to what might reasonably be expected (also according to evidence at the micro-level) in terms of rates of returns, many studies' estimates are way too high (see Button, page 153).

Even though these points are all well taken and should be kept in mind as caveats, we are still convinced that it is of importance to pursue the topic, since if there is any conclusion to be drawn from the existing literature it is that there is a significant relationship between GDP and infrastructure (no matter what the precise nature is) and that this is highly policy-relevant from a development perspective and also in terms of the efficiency of ongoing production processes (i.e. infrastructure is not only an independent input into the production process, but also has an indirect effect on economic growth by enhancing the marginal products of other forms of capital, labor, energy and materials). Let us thus first begin with a definition of what infrastructure means in the context of our work and how we like to place our contribution within the range of the existing literature.

Button (1998) lists in his review a number of definitions of infrastructure, which range from very specific to highly aggregate and thus also quite vague notions sometimes. The one that appears most inclusive, yet precise, to us is the one by Hirschman (1958): "[...] it includes all public services from law and order through education and public health to transportation, communications, power and water supply as well as agricultural overhead capital as irrigation and drainage systems. The core of the concept can probably be restricted to transportation and power." (Button, page 150). We agree largely with this definition and have decided to focus on the first type of these "core" infrastructure, which is transportation, for our application. In particular, we concentrate our analysis on road infrastructures, which is of course an arbitrary choice. However, our main purpose is not to conduct another empirical study, but rather to introduce a new modeling approach, so our contribution is more on the theoretical side and all empirical implementations have only been conducted for the sake of demonstration and verification of applicability and usefulness for real world analysis. It is thus possible to use the method for any other type of infrastructure as well, given that the relationships between the variables in the model are adapted to the new infrastructure context.

Button (1998) distinguishes in his review of the infrastructure and growth literature between two "schools", where the first one is referred to as the Keynesian approach, which starts from the notion that any income or infrastructure can only be generated by economic growth itself in the first place. The other school is that of the neo-classical approach, which treat infrastructure as a production factor in the same style as labor and capital and which belong mainly to the literature of endogenous growth modeling. Fedderke et al (2006), for example, carry out a time-series analysis for investment into road infrastructure and economic growth in South Africa and find that the former does indeed lead to economic growth in South Africa, both by boosting GDP directly and by raising the marginal products of other production factors. They also test for the other direction of causality (i.e. from GDP growth to infrastructure expansion), but the evidence is significantly weaker in this case.

It is not entirely clear to which "camp" our approach developed here belongs: on the one hand, we develop a model, where the amount of newly added infrastructure is the control, which we optimize to foster growth and eventually reach a steady state. On the other hand, we adopt a co-evolutionary perspective taking on the view that the level GDP and the stock of infrastructure develop simultaneously, thereby enhancing each other. In any case, we abstract from major secondary effects, such as pointed out by Button (1994), who claims that road infrastructure will not benefit regions, where the new roads simply serve transit traffic, or where producers do not have a comparative advantage over their newly accessible trade partners. Since our study takes the point-of-view of the social planner 
at a more aggregate level, such considerations, which matter for the distribution of gains from infrastructure expansion within the country, are first neglected, even though we do not want to downplay their importance for further research.

Coming back to the issue of policy relevance, many of the empirical studies conducted so far have been motivated by the need to form policy recommendations targeted at exploiting the potential of infrastructure to foster economic growth. Liberini (2006), for example, employs an econometric approach to estimate the so-called "infrastructure gap", which is defined as the difference between infrastructure demand based on potential GDP 3 and the level of infrastructure that is actually provided. The aim of Liberini's study is to determine the impact that government retrenchment on public funding of infrastructure had in the Latin American countries that were also affected by the debt crisis and to compare these results with infrastructure gaps computed for other regions like the OECD and East Asia, as far as data availability permits. Her reasoning with respect to the infrastructure gap provides a justification for our approach to set up a co-evolutionary model: in Liberini's (2006) framework, public infrastructure investment ${ }^{4}$ exceeding the optimum evokes a negative correlation between economic growth and infrastructure expansion and vice versa, so that the idea that GDP and infrastructure co-evolve and are drawn towards a steady state (either from below or from above) appears quite intuitive. Liberini's (2006) findings indicate a positive elasticity of infrastructure with respect to per capita GDP with respect to telecommunications and road capacity. Furthermore, she tests for the significance of infrastructure quality indices, which are found to be negative, implying that the existence of higher quality infrastructure will reduce the need for further expansions in the short term. In our study we also investigate the importance of quality differences modeled through variations in the rate of physical decay of roads. As regards the infrastructure gap, Liberini (2006) concludes that it has been increasing during the period of the debt crisis in many Latin American countries (and in most sectors), while East Asia seems to have maintained their gap at a stable level (and if better data were available, this would improve the results for East Asia even more, as some well-performing countries are not accounted for in the available data set used in the study). OECD countries can generally be reported with constant or even shrinking gaps. These results hold for telecommnunications and power; however, in the case of road infrastructure a decrease in infrastructure gaps can be observed across all regions, which might point to the fundamentality of road infrastructure compared to other types of infrastructure and thus further justifies our choice to concentrate on roads in this paper as well.

Our contribution is more of a theoretical nature, even though we also apply the developed framework to data from some OECD countries $^{5}$ in order to show that the approach can also be useful to gain insight into real world situations or in order to derive policy recommendations given specific conditions hold. Our results show that we can derive an analytical solution to the problem of optimal infrastructure expansion, for some prespecified functional relationships between GDP, maintenance and investment costs and existing stocks and changes in stocks of infrastructure, ${ }^{6}$ if the control is kept constant. We find an analytical solution for the infinite horizon problem, where the control turns out

\footnotetext{
${ }^{3}$ Potential GDP is that level of output that could be produced if all production factors could be used to their fullest extent.

${ }^{4}$ Liberini (2006) also mentions that private investment in infrastructure was not sufficient to counterbalance the retrenchment of public funds in Latin American countries. Since we take an aggregate view of the problem, we refrain from an explicit distinction between private and public investment as well.

${ }^{5}$ We present the cases of Finland and France here for illustrative purposes.

${ }^{6}$ These functional relationship can of course be changed, should the particular circumstances and characteristics of a country require so. We have here tried to come up with the most basic and intuitive reasoning to illustrate the usefulness of the co-evolutionary and optimal control approach.
} 
to be a constant. The steady state is shown to depend crucially on the rate of physical decay of roads, which we think can be interpreted as an index of quality, and the speed of adjustment, at which the economy moves along a trajectory. Testing the model for the data of two countries, France and Finland, illustrates the usefulness of such an approach to real world problems and possibly policy recommendation, where the model would have to be adapted to the peculiarities of each country or region to make precise statements. For the (more impressionistic) country studies presented here, both France and Finland are below their steady states, although France is rather close to it, while Finland is relatively farther removed. An increase in quality modeled through lower depreciation of the existing infrastructure stock is shown to lead to a higher steady state, which implies that a higher level of GDP can be reached in the long run. In this context, another important insight is the dependence of the results on the parametrization, in particular the tradeoff between the speed of adjustment, with which GDP approaches the asymptote, and the rate of decay of the existing road stock, as mentioned before.

The paper is organized as follows. In Sections 2 the optimal control model is developed, motivated by a co-evolutionary perspective on the interactive development of infrastructure and GDP. We manage to solve the model analytically for the long run, i.e. when the planning horizon is infinite. The finite horizon case is presented in the Appendix A. Section 3 gives an overview of the methods used to calibrate the core equations presented in the previous section. The results for two exemplary countries are presented and briefly discussed in Section 4. Section 5 summarizes the findings of this study, discusses their relevance and gives an outlook to future research in this area.

\section{Optimal Control Approach to Infrastructure Investment \& Economic Growth}

\section{$2.1 \quad$ Model}

The model presented here is essentially based on the assumption that there is a strong interdependency between the capacity of transportation infrastructure and economic growth. Adopting this assumption we introduce a model of co-evolutionary dynamics that qualitatively describes how the development of transportation infrastructure affects the rate of economic growth and vice versa. The qualitative co-evolutionary model is in turn used to construct a control model of development of transportation infrastructure in the context of economic growth.

We assume that the capacity of the country's road infrastructure, $z$, creates a basis for the country's GDP growth and introduce the threshold function $f(z)$ that characterizes the maximal possible level of GDP provided by a given road capacity, $z$. If the current level of GDP, $x$, is below the baseline, $x<f(z)$, then the GDP grows. If the level of GDP is above the baseline, the GDP decreases. Symmetrically, we assume that the level of a country's GDP, $x$, determines the development of the country's road infrastructure and introduce the threshold function $h(x)$ characterizing the size of the road capacity that can be supported by a given level of GDP, $x$. If the current level of GDP is too low for the existing road capacity, $z>h(x)$, then the size of road capacity decreases due to physical decay, as there is not sufficient investment to support the stock of road infrastructure. Conversely, if the current level of GDP can support the greater size of road capacity, then the capacity increases. Obviously, $f(z)$ and $h(x)$ are monotonically increasing functions.

Figure 1 shows how the phase diagram corresponding to the co-evolutionary model looks like. The threshold functions split the diagram into three regions: above the baseline $f(z)$, between the baselines $f(z), h(x)$, and below the baseline $h(x)$. For each region, the 


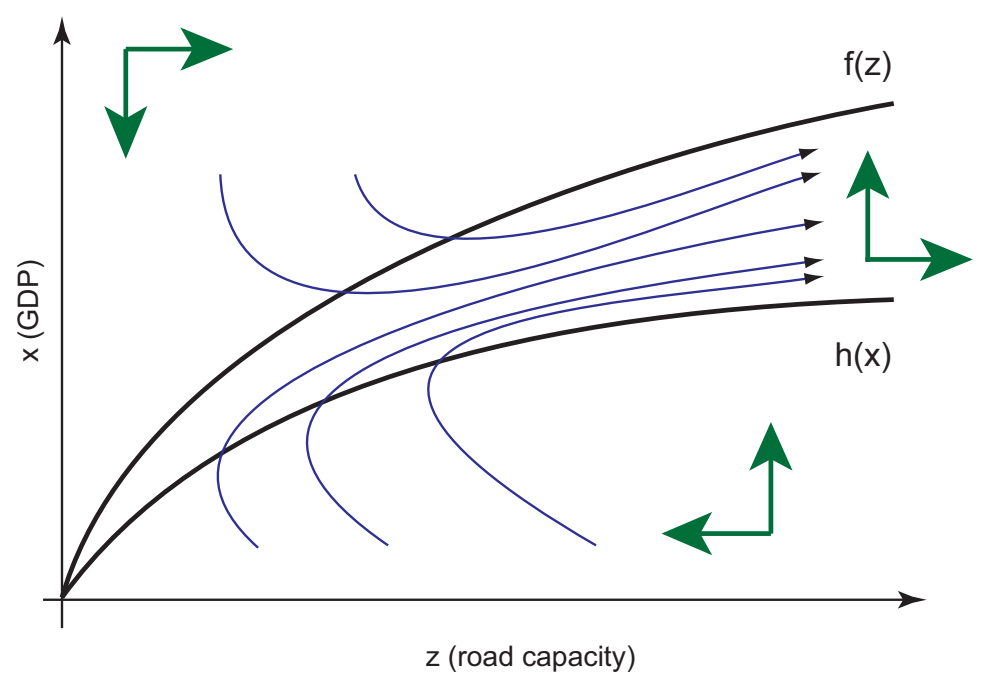

Figure 1: Co-evolutionary dynamics

directions of change for the road capacity and the level of GDP are indicated.

Based on the co-evolutionary model described, we construct a control-theoretic model of the development of road infrastructure. Assuming road capacity, $z$, to change over time, we get

$$
\dot{z}(t)=u(t)-\delta z(t) .
$$

Here $z(t)$ is the road capacity at time $t, u(t)$ is its growth rate at time $t$ and $\delta$ is the depreciation rate. We set

$$
\begin{aligned}
& z(0)=z^{0}, \\
& 0 \leq u(t) \leq \bar{u},
\end{aligned}
$$

where $z^{0}$ is the road capacity at the initial time, 0 , and $\bar{u}$ is the maximal possible growth rate of the road capacity. Assuming the level of a country's annual GDP, $x$, to depend on road capacity and that the maximal possible level of GDP provided by existing road capacity, $z$, is determined by the threshold function $f(z)$, we get

$$
\dot{x}(t)=\gamma(f(z(t))-x(t)),
$$

where $x(t)$ is the level of GDP at time $t$ and $\gamma$ is a coefficient of the speed of adjustment. We set

$$
x(0)=x_{0},
$$

where $x^{0}$ is the initial level of GDP.

Let $c(z)$ be the annual cost of maintaining road capacity $z$ and $r(u)$ be the cost of increasing road capacity by an amount $u$ in one year. Obviously, $c(z)$ and $r(u)$ are again monotonically increasing functions. It is reasonable to assume that $c$ and $r$ go to infinity as $z$ and $u$ do. The country's annual benefit is given by

$$
b(z, x, u)=\mu x-c(z)-r(u),
$$

where $\mu$ is the portion of GDP composed of road infrastructure. In the Section 2.3 there will be further explanations for how $\mu$ can be calibrated and in what range we can expect 
this parameter to lie. Assuming an integrated benefit discounted at rate $\rho$,

$$
J=\int_{0}^{\infty} e^{-\rho t} b(z(t), x(t), u(t)) d t
$$

to be the country's utility, we end up with an optimal control problem: ${ }^{7}$

$$
\begin{array}{ll}
\text { maximize } & J=\int_{0}^{\infty} e^{-\rho t} b(z(t), x(t), u(t)) d t \\
\text { subject to } & (1)-(5) .
\end{array}
$$

\subsection{Specifying Functions}

The most coherent data set with the longest cross-country time series for road length and other indicators for road infrastructure was compiled by Canning $(1998,1999)$. Figures 2 to 4 below display these data plotted against GDP, starting with road length in kilometers, with some data also taken from the European Conference of Ministers of Transportation (1998). It is evident that the relationship is positive and in most cases close to linear. The other two Figures show road traffic (in millions of vehicle kilometers) and road energy consumption (in tons of oil equivalent) against GDP respectively. These relationships confirm the previous observations.

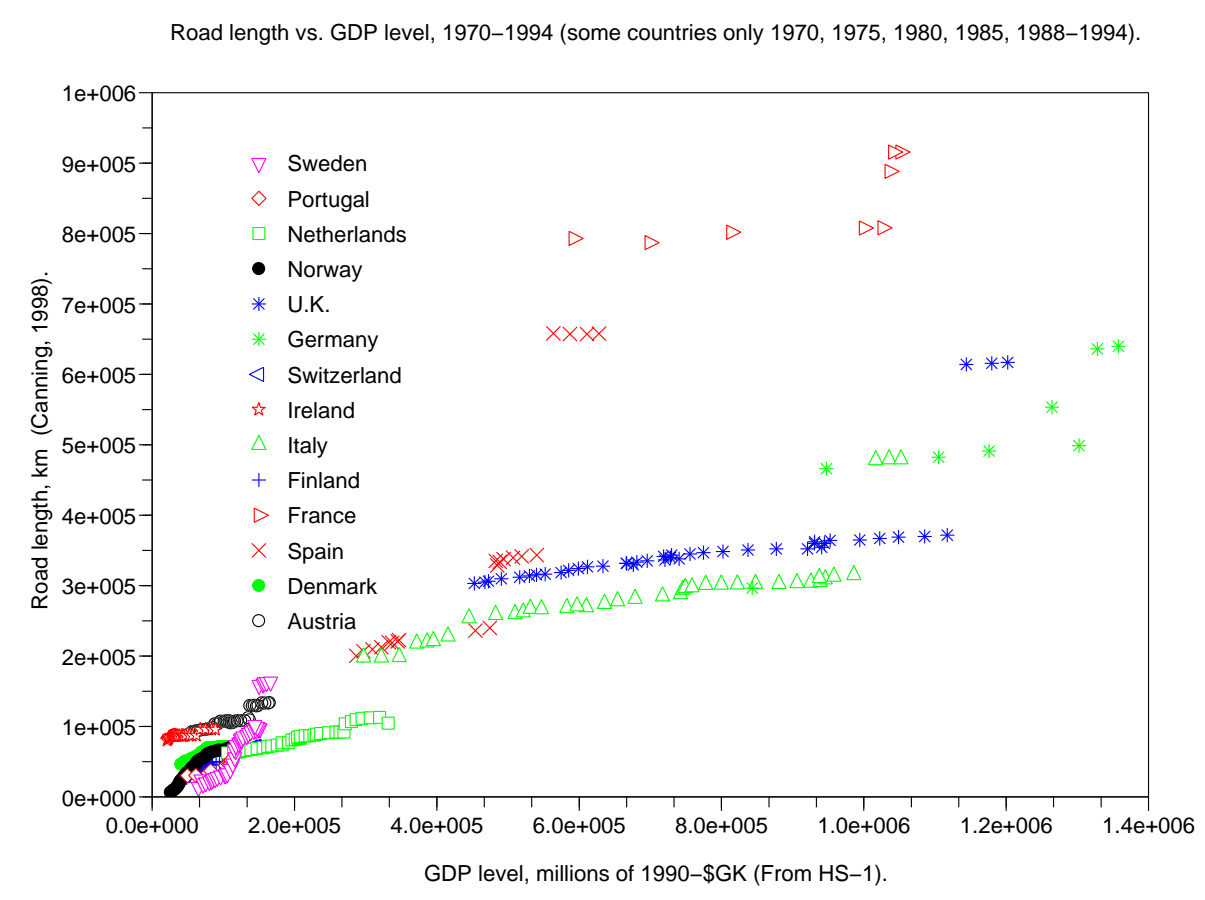

Figure 2: Road length (in $\mathrm{km}$ ) against the level of total GDP (in mill. 1990 GK\$)

Here we specify the functions needed for the control model: the threshold function, $f(z)$, for the country's GDP depending on the existing capacity of road infrastructure; the cost of expanding the infrastructure, $r(u)$, depending on the level of investment into new infrastructure; and the cost of maintaining the infrastructure, $c(z)$.

\footnotetext{
${ }^{7}$ We want to refer the reader to the appendix for the problem with a finite planning horizon.
} 
Road traffic vs. GDP levels (1970, 1975, 1980, 1985, 1988-1994.)

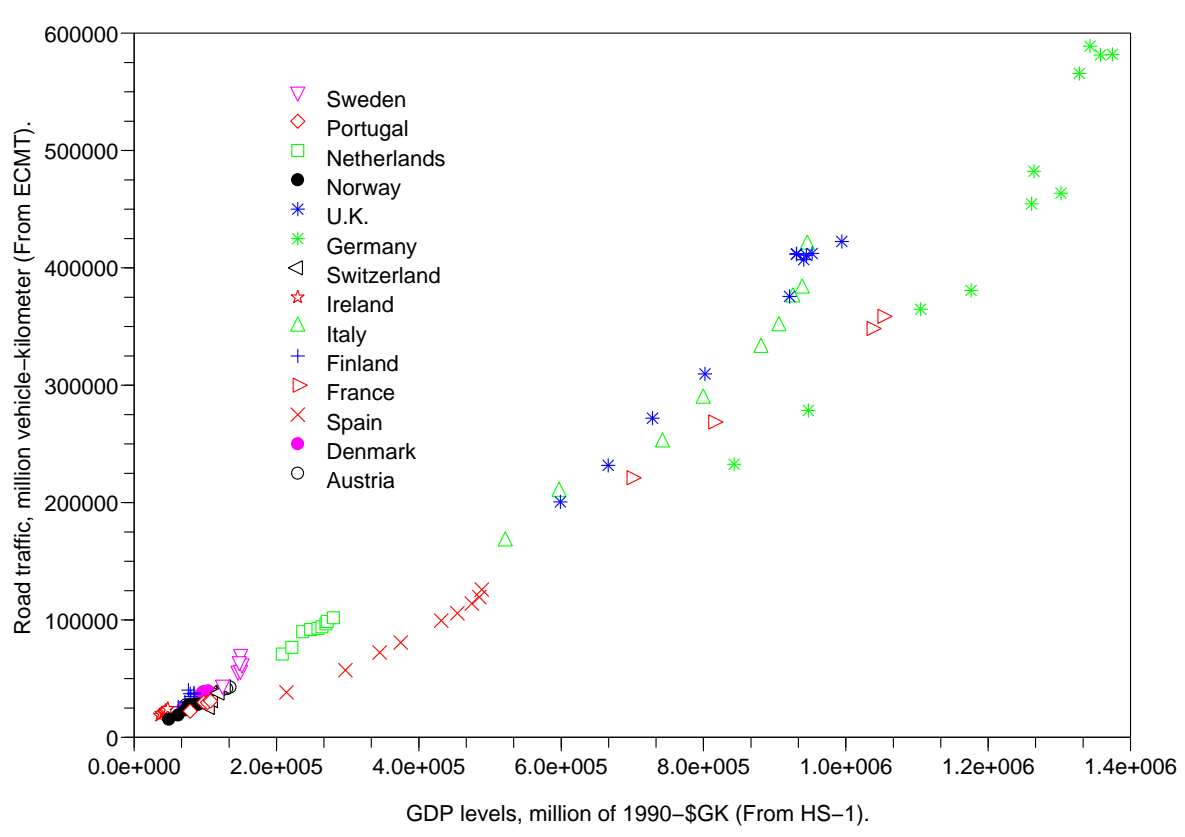

Figure 3: Road traffic (in mill. vehicle $\mathrm{km}$ ) against the level of total GDP (in mill. 1990 $G K \$)$

Energy consumption of the road transport sector vs. GDP level (1970, 1975, 1980, 1985, 1988-1994)

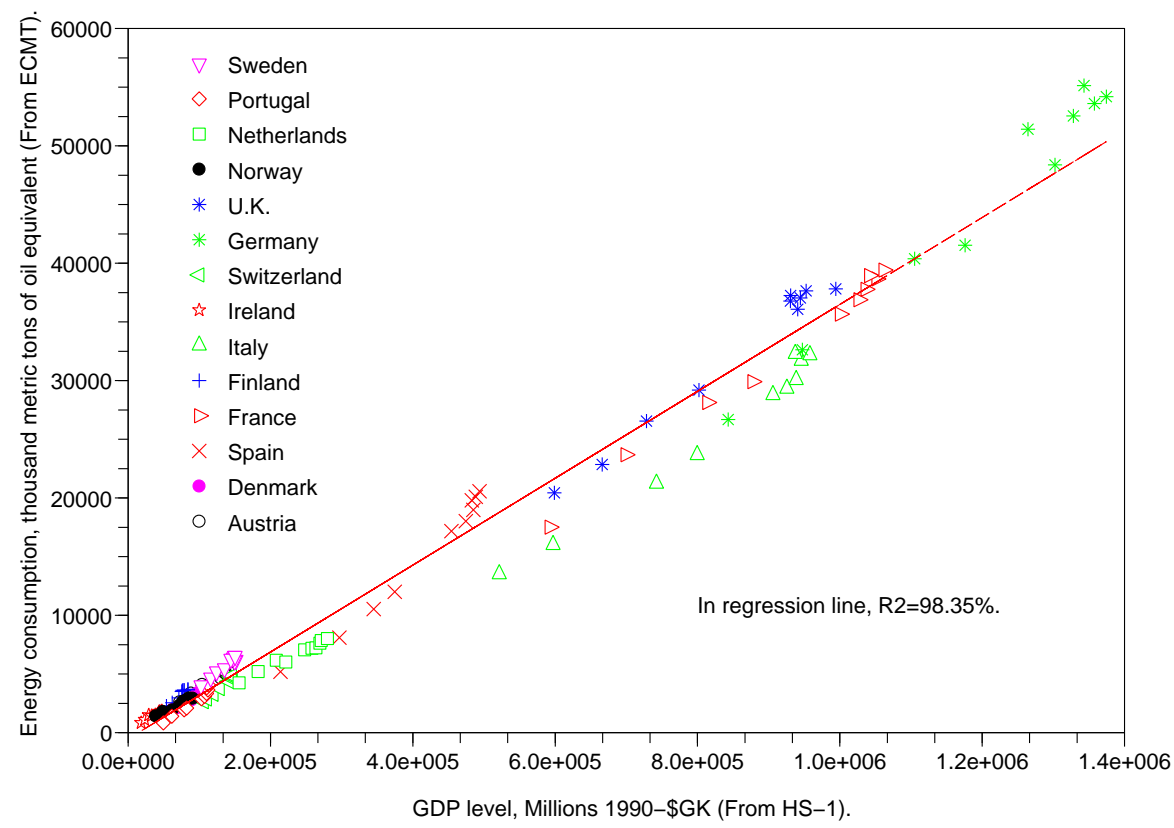

Figure 4: Road transport energy consumption (in tons of oil equivalent) against the level of total GDP (in mill. 1990 GK\$) 
1. Denote by $g(z)$ the function for the country's GDP, depending on the existing capacity of road infrastructure. This function is introduced to reflect the dependency between the country's GDP and the capacity of infrastructure based on statistical data. Looking at Figures $2-4$, we assume that it is a linear function, so

$$
g(z)=\alpha z+\beta .
$$

The coefficients $\alpha$ and $\beta$ will be calibrated for every country separately using statistical methods.

2. The threshold $f(z)$ is assumed to be a linear function

$$
f(z)=A z+B
$$

3. In order to specify the function of the cost of investing in infrastructure, $r(u)$, we make the reasonable assumption that small amendments to existing infrastructure are relatively inexpensive, while setting up a major, new capacity item or a whole infrastructure system in the first place is much more costly. Moreover, we impose a restriction that no investment must also imply no cost, i.e. $r(0)=0$. Hence, we infer that $r(u)$ is an exponential function:

$$
r(u)=L e^{\theta u}-L,
$$

where $L$ and $\theta$ are positive constants.

4. Considering an example of maintaining (e.g. through repairing) roads, we assume that the maintenance cost as a function of existing infrastructure is a linear function

$$
c(z)=D z+E,
$$

where $D$ and $E$ are constants.

\subsection{The Share of Road Infrastructure in Economic Output}

The parameter $\mu$ is the portion of the GDP which can be attributed to road infrastructure and so it can be interpreted as the importance of the role that road infrastructure plays in total economic output, the other contributing factors being labor, resources, other types of physical capital, energy, human capital and so forth. In the country case studies presented in the later sections, we have used a value of $5 \%$ as an - admittedly cautious - benchmark, since we did not want to overstate the effect of road infrastructure on total GDP in the face of relatively little constraints on that relationship. Table 1 shows, however, that $\mu$ could potentially be higher than that.

The data on total energy consumption are from BP p.l.c. (Statistical Review of World Energy, 2008), while the data on energy consumption in the road sector was taken from Maddison (2001). It is necessary to estimate the ratio $\mu$ of GDP output, which is attributable to the activity in the road transportation sector. To this end we use the plot in Figure 4, which shows a linear relation between countries' total GDP and energy consumption of the road sector as well as Table 1, which shows the ratio of energy used in the road sector to total energy consumption. This ratio displays an increasing trend; the last available values, corresponding to the year 1994, are between $7.6 \%$ for Norway to $22.5 \%$ for Portugal. Some estimates say that the transport industry is responsible for producing $6-8 \%$ of GDP in most countries (Weidlich et al, 1999), in the case of France a more precise 


\begin{tabular}{lcccccccc}
\hline Country & 1980 & 1985 & 1989 & 1990 & 1991 & 1992 & 1993 & 1994 \\
\hline Austria & 0.154 & 0.154 & 0.168 & 0.168 & 0.177 & 0.180 & 0.174 & 0.182 \\
Switzerland & 0.131 & 0.142 & 0.168 & 0.168 & 0.170 & 0.171 & - & - \\
Germany & 0.113 & 0.115 & 0.136 & 0.147 & 0.154 & 0.159 & 0.165 & 0.163 \\
Denmark & 0.117 & 0.151 & 0.182 & 0.187 & 0.169 & 0.187 & 0.176 & 0.172 \\
Spain & 0.137 & 0.153 & 0.190 & 0.198 & 0.204 & 0.211 & 0.210 & 0.212 \\
France & 0.147 & 0.152 & 0.167 & 0.168 & 0.163 & 0.165 & 0.166 & 0.171 \\
Finland & 0.120 & 0.133 & 0.155 & 0.159 & 0.150 & 0.154 & 0.151 & 0.149 \\
Italy & 0.147 & 0.171 & 0.187 & 0.189 & 0.190 & 0.200 & 0.208 & 0.207 \\
Ireland & 0.197 & 0.199 & 0.181 & 0.180 & 0.177 & 0.192 & 0.185 & 0.184 \\
Norway & 0.065 & 0.067 & 0.069 & 0.067 & 0.076 & 0.073 & 0.074 & 0.076 \\
Netherlands & 0.083 & 0.086 & 0.095 & 0.094 & 0.091 & 0.097 & 0.098 & 0.100 \\
Portugal & 0.176 & 0.174 & 0.191 & 0.193 & 0.202 & 0.214 & 0.221 & 0.225 \\
Sweden & 0.113 & 0.107 & 0.130 & 0.121 & 0.122 & 0.126 & 0.125 & 0.131 \\
U. K. & 0.131 & 0.144 & 0.171 & 0.175 & 0.170 & 0.173 & 0.173 & 0.177 \\
\hline
\end{tabular}

Table 1: Ratio of energy used in road sector to total energy consumption

evaluation states that the transport industry share of GDP is around 14\% (French Road Federation, 2006).

Since these estimates display a large range of diverse numbers, we have decided to keep $\mu$ low at around $5 \%$ for the beginning, as we want to avoid overstating the effects of a larger stock of road infrastructure on steady state GDP in the absence of strict constraints on that relationship. In the case studies presented in Section 4 the sensitivity of the results with respect to higher values of $\mu$ will be tested.

\subsection{Solution of Optimal Control Problem}

In this section, we approach the problem from an optimal control point-of-view (e.g. Pontryagin et al (1962); Lee and Marcus (1967); see Dorfman (1969) for a more economic exposition of optimal control problems).

We consider the following optimal control problem with infinite time horizon

$$
\begin{aligned}
\text { maximize } J & =\int_{0}^{\infty} e^{-\rho t}\left(\mu x(t)-D z(t)-E-L e^{\theta u(t)}+L\right) d t \\
\text { subject to } & \\
\dot{z}(t) & =u(t)-\delta z(t), \\
\dot{x}(t) & =\gamma(A z(t)+B-x(t)), \\
u(t) & \in[0, \bar{u}] \\
z(0) & =z_{0}, \\
x(0) & =x_{0}, \\
t & \in[0, \infty) .
\end{aligned}
$$

An approach to find a solution of the problem is based on the Pontryagin Maximum Principle for a case of infinite time horizon. More precisely, we use Corollary 7 proved in Aseev and Kryazhimskiy (2005). First, let us check that the problem satisfies a number of assumptions in order to prove the applicability of the method to the problem. 
Assumption 1 [A3 $]^{8}$. For each $z$ and each $x$, the function $b(z, x, u)$ is a concave function in $u$.

That follows from the convexity of the exponential function $e^{\theta u}$ and the condition $L>0$.

Assumption 2 [A4]. There exist positive-valued functions $\mu$ and $\omega$ on $[0, \infty)$ such that $\mu(t) \rightarrow 0$ as $t \rightarrow \infty$, and for any admissible pair $(u, z, x)$,

$$
\begin{gathered}
e^{-\rho t} \max _{u \in[0, \bar{u}]}|b(z(t), x(t), u)| \leq \mu(t) \text { for all } t>0 ; \\
\int_{T}^{\infty} e^{-\rho t}|b(z(t), x(t), u(t))| d t \leq \omega(T) \text { for all } T>0 .
\end{gathered}
$$

That follows from the linearity of the function $b(z, x, u)$ in $(z, x)$, and the restrictions on control function.

Assumption 3 [A6]. There exists $a k \geq 0$ and $a r \geq 0$ such that

$$
\sqrt{\left(\frac{\partial b(z, x, u)}{\partial z}\right)^{2}+\left(\frac{\partial b(z, x, u)}{\partial x}\right)^{2}} \leq k\left(1+\sqrt{z^{2}+x^{2}}\right)^{r} \text { for all } x \text { and for all } u \in[0, \bar{u}] \text {. }
$$

Taking into account the linearity of the function $b(z, x, u)$ in $(z, x)$, we get that $k=$ $\sqrt{\mu^{2}+D^{2}}$ and $r=0$.

\section{Assumption 4 [Dominating discount case].}

$$
\rho>(r+1) \lambda,
$$

where $\lambda$ is the maximal of the real parts of the eigenvalues of the dynamic system.

Taking into account that $r=0$ and all eigenvalues of the dynamic system are negative we get that it is sufficient that $\rho>0$.

Now we can start solving the problem using the Maximum principle. Let us compose the Hamilton-Pontryagin function

$$
\mathcal{H}(t, z, x, u, \psi)=e^{-\rho t}\left(\mu x-D z-E-L e^{\theta u}+L\right)+\psi_{1}(u-\delta z)+\psi_{2} \gamma(A z+B-x)
$$

and the adjoint equation

$$
\left\{\begin{array}{l}
\dot{\psi}_{1}=-\frac{\partial \mathcal{H}}{\partial z}=\delta \psi_{1}-\gamma A \psi_{2}+D e^{-\rho t} \\
\dot{\psi}_{2}=-\frac{\partial \mathcal{H}}{\partial x}=\gamma \psi_{2}-\mu e^{-\rho t}
\end{array}\right.
$$

Using Corollary 7 (Aseev and Kryazhimskiy (2005)), we get the following transversality condition

$$
\begin{aligned}
& \lim _{t \rightarrow \infty} \psi_{1}(t)=0, \\
& \lim _{t \rightarrow \infty} \psi_{2}(t)=0 .
\end{aligned}
$$

Let us consider the differential equation describing the adjoint variable $\psi_{2}$ separately

$$
\dot{\psi}_{2}=\gamma \psi_{2}-\mu e^{-\rho t}
$$

A general integral of this equation has the following form

$$
\psi_{2}(t)=\frac{\mu}{\rho+\gamma} e^{-\rho t}+C_{1} e^{\gamma t}
$$

\footnotetext{
${ }^{8}$ Numbers in square brackets refer to the assumptions in Aseev and Kryazhimskiy (2005).
} 
where $C_{1}$ is a constant. Taking into account transversality condition (16), we get

$$
C_{1}=0 .
$$

Hence, we have

$$
\psi_{2}(t)=\frac{\mu}{\rho+\gamma} e^{-\rho t}
$$

Substitute the variable $\psi_{2}$ in the differential equation for the variable $\psi_{1}$ using the equality (18). We get the following differential equation for the variable $\psi_{1}$

$$
\dot{\psi}_{1}=\delta \psi_{1}-\left(\frac{\gamma A \mu}{\rho+\gamma}-D\right) e^{-\rho t}
$$

This equation has the following general integral

$$
\psi_{1}(t)=\frac{\gamma A \mu-D \gamma-D \rho}{(\gamma+\rho)(\delta+\rho)} e^{-\rho t}+C_{2} e^{\delta t} .
$$

Taking into account the transversality condition (17), we get

$$
\psi_{1}(t)=\frac{\gamma A \mu-D \gamma-D \rho}{(\gamma+\rho)(\delta+\rho)} e^{-\rho t} .
$$

The extremal control satisfies the following maximum condition

$$
u(t, z, x, \psi)=\underset{u \in[0, \bar{u}]}{\arg \max } \mathcal{H}(t, z, x, u, \psi)=\underset{u \in[0, \bar{u}]}{\arg \max }\left\{\psi_{1} u-L e^{-\rho t} e^{\theta u}\right\} .
$$

Let us note, that the function

$$
M(u)=\psi_{1} u-L e^{-\rho t} e^{\theta u}
$$

is a concave function as $L>0$. Therefore,

$$
\underset{u \in[0, \bar{u}]}{\arg \max } M(u)= \begin{cases}0, & \hat{u}(t) \leq 0, \\ \hat{u}(t), & \hat{u}(t) \in(0, \bar{u}), \\ \bar{u}, & \hat{u}(t) \geq \bar{u},\end{cases}
$$

where $\hat{u}$ is a solution of the equation

$$
\frac{\partial M(u)}{\partial u}=0 .
$$

We get

$$
\hat{u}(t)=\frac{1}{\theta} \ln \left(\frac{e^{\rho t} \psi_{1}(t)}{L \theta}\right) .
$$

Finally, we get the optimal control

$$
u^{*}(t)=\left\{\begin{array}{l}
0, \quad \hat{u} \leq 0 \\
\hat{u}, \quad \hat{u} \in(0, \bar{u}) \\
\bar{u}, \quad \hat{u} \geq \bar{u}
\end{array}\right.
$$

where

$$
\hat{u}=\frac{1}{\theta} \ln \left(\frac{\gamma A \mu-D \gamma-D \rho}{L \theta(\gamma+\rho)(\delta+\rho)}\right) .
$$




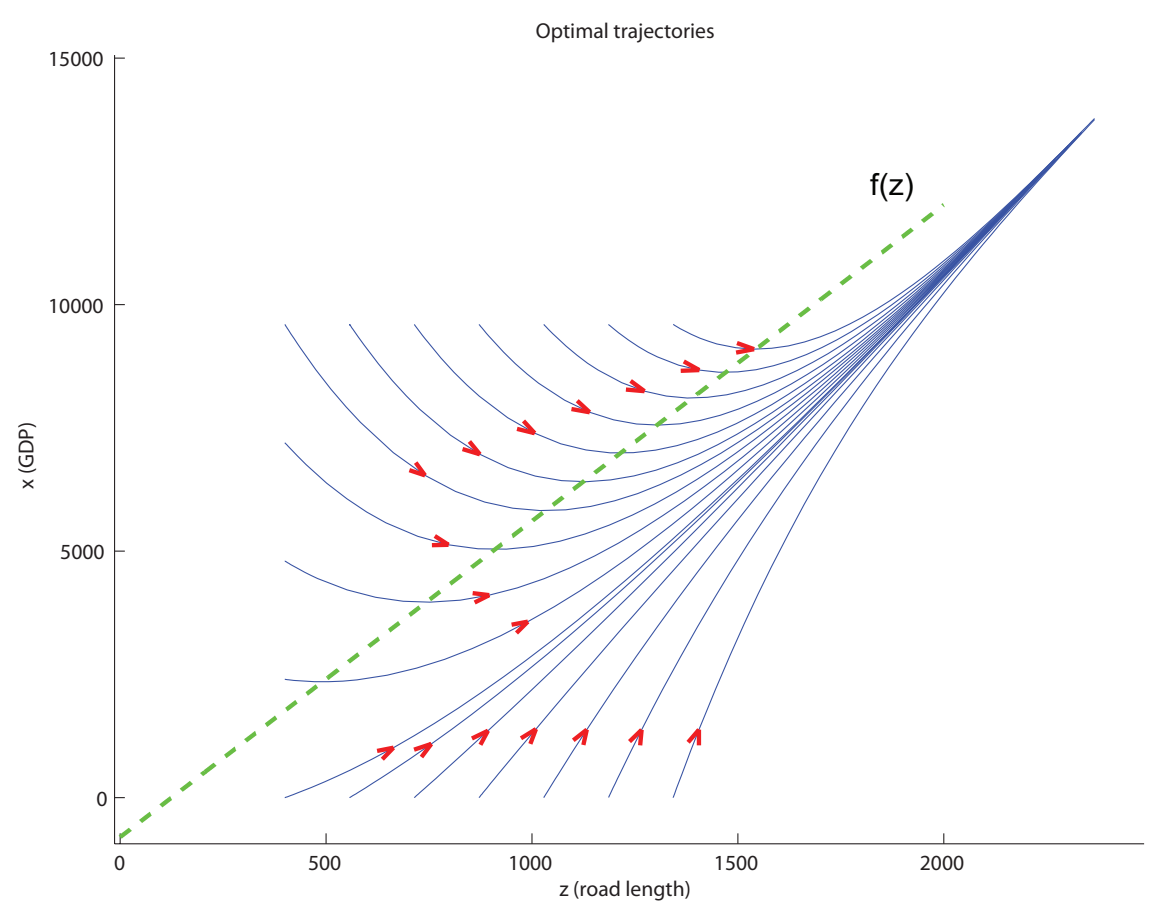

Figure 5: Optimal trajectories starting at various initial states and the threshold curve $f(z)$ (green color).

An important conclusion is that the optimal control $u^{*}(t)$ is a constant function over time interval $t \in[0, \infty)$. Figure 5 shows an example of a phase diagram that consists of a number of optimal trajectories starting at various initial states. The threshold curve corresponding to the function $f(z)$ has a lighter shade (green in color version). Trajectories starting above the threshold line go down, that is GDP decreases, until a trajectory intersects the threshold line, and after that GDP increases. Later, we will show that the trajectories' behavior not only depends on the threshold line but on the steady state as well, which uniquely exists for every optimal trajectory in the model.

Let us describe how the second threshold function, $h(x)$, affects the phase diagram. We introduced a constant restriction on the control, $0 \leq u \leq \bar{u}$. However, the upper restriction actually depends on the current level of GDP because we are not able to invest much money if the level of GDP is low, so the upper restriction looks like $u \leq \bar{u}(x)$. Therefore, there is a possibility that the size of road capacity can decrease due to physical decay, while the level of GDP is low. Consequently, the function $\bar{u}(x)$ determines a threshold curve, denoted by $h(x)$, that separates areas of decreasing and increasing road capacity size. Figure 6 presents a phase diagram with an upper restriction on the control $0 \leq u \leq \bar{u}(x)$. In the present paper, we will not be considering such kinds of restrictions on the control, as we have no information to calibrate the function $\bar{u}(x)$ or $h(x)$.

Let us substitute the constant control $u^{*}$ into the equations describing the dynamical system. The equations take the form

$$
\begin{cases}\dot{z}(t)=u^{*}-\delta z(t), & z(0)=z_{0}, \\ \dot{x}(t)=\gamma(A z(t)+B-x(t)), & x(0)=x_{0} .\end{cases}
$$

That means that, assuming $u=u^{*}$, the trajectory of the system can be computed as the 


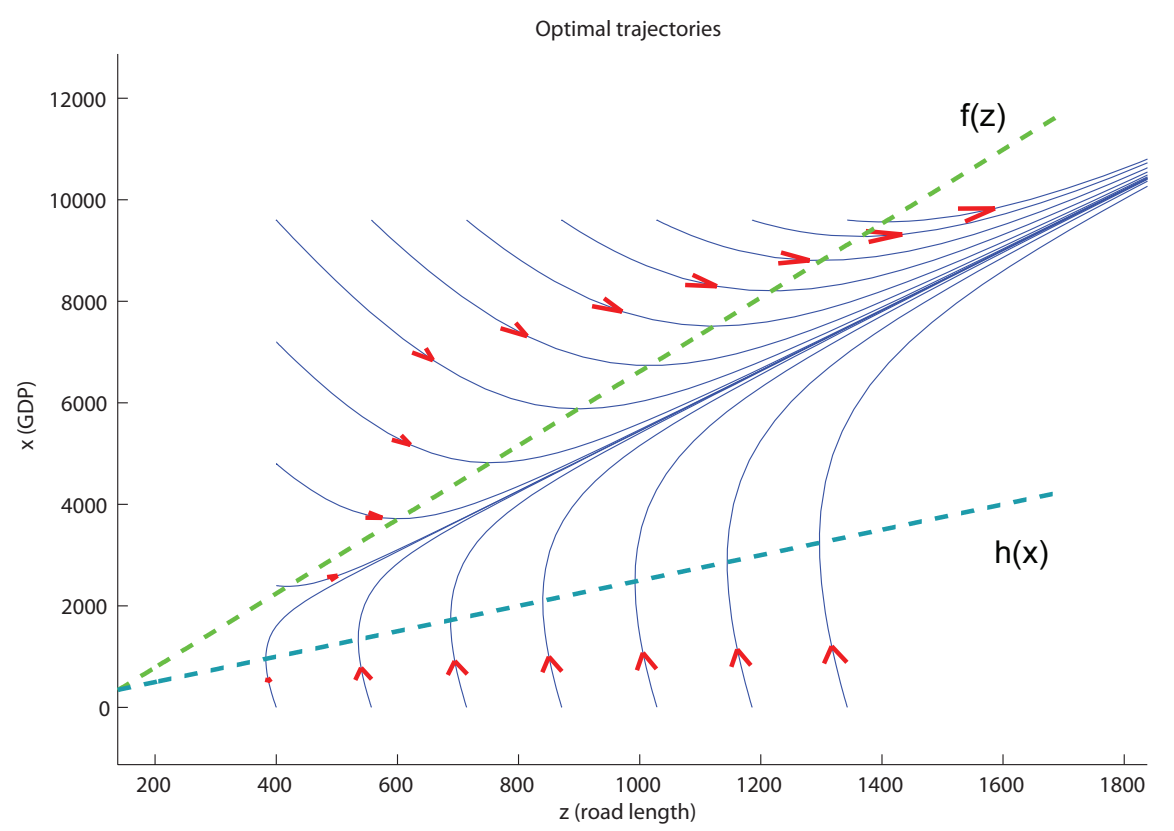

Figure 6: Optimal trajectories and the threshold curves $f(z)$ (green color) and $h(x)$ (blue color).

solution of an affine system

$$
\dot{y}=F y+G,
$$

where

$$
y=\left(\begin{array}{c}
z \\
x
\end{array}\right), \quad F=\left(\begin{array}{cc}
-\delta & 0 \\
\gamma A & -\gamma
\end{array}\right), \quad G=\left(\begin{array}{c}
u^{*} \\
\gamma B
\end{array}\right)
$$

satisfying the initial condition

$$
y(0)=\left(\begin{array}{c}
z_{0} \\
x_{0}
\end{array}\right) .
$$

The solution of the system can be calculated analytically as

$$
\left\{\begin{array}{l}
z(t)=-\frac{u^{*}-\delta z_{0}}{\delta} e^{-\delta t}+\frac{u^{*}}{\delta} \\
x(t)=\gamma A \frac{u^{*}-\delta z_{0}}{\delta(\delta-\gamma)} e^{-\delta t}+\left(x_{0}-B+\delta A \frac{\gamma z_{0}-u^{*}}{\delta(\delta-\gamma)}\right) e^{-\gamma t}+\frac{B \delta+A u^{*}}{\delta}
\end{array}\right.
$$

for $\delta \neq \gamma$ and

$$
\left\{\begin{array}{l}
z(t)=-\frac{u^{*}-\delta z_{0}}{\delta} e^{-\delta t}+\frac{u^{*}}{\delta} \\
x(t)=A\left(\delta z_{0}-u^{*}\right) t e^{-\delta t}+\left(x_{0}-A \frac{u^{*}}{\delta}-B\right) e^{-\delta t}+\left(A \frac{u^{*}}{\delta}+B\right)
\end{array}\right.
$$

for $\delta=\gamma$. Since the eigenvalues of $F$ are $-\gamma,-\delta$, that means both are negative, the unique stationary solution

$$
\hat{y}=\left(\begin{array}{c}
\hat{z} \\
\hat{x}
\end{array}\right)=\left(\begin{array}{c}
\frac{u^{*}}{\delta} \\
A \hat{z}+B
\end{array}\right)
$$


of the system is a stable node and the trajectories converge to it along the eigenvector belonging to the eigenvalue closer to zero for $\delta \neq \gamma$. That means that for $\delta<\gamma$ the trajectories converge along the line

$$
x=\frac{\gamma A}{\gamma-\delta} z-A \frac{u^{*}}{\gamma-\delta}+B
$$

and for $\delta>\gamma$ along the line

$$
z=\frac{u^{*}}{\delta} .
$$

Let us assume that the developed countries already behave optimally (that means their observed real trajectory follows the asymptote whose eigenvector has a smaller modulus). Therefore, the asymptotic line computed for a developed country must coincide with the function $g(z)$ calibrated for the same country. We use this assumption to calibrate the threshold function $f(z)$. We get two equations to compute $A$ and $B$

$$
\left\{\begin{array}{l}
\alpha=\frac{\gamma A}{\gamma-\delta}, \\
\beta=-A \frac{u^{*}}{\gamma-\delta}+B .
\end{array}\right.
$$

Solving the latter equations, we get

$$
\left\{\begin{array}{l}
A=\frac{\alpha(\gamma-\delta)}{\gamma}, \\
B=\beta+\frac{\alpha}{\gamma} u^{*} .
\end{array}\right.
$$

Figure 7 presents an example of two asymptotic lines with a darker shade (highlighted in magenta in the color version). All optimal trajectories converge along the inclined asymptotic line in the case of $\gamma>\delta$ and along the vertical asymptotic line in the case of $\gamma<\delta$, and terminate in a unique steady state.

The steady state is an essential element of the optimal behavior of the described control model. It gives the maximal possible level of GDP and the maximal possible road capacity to support that level of GDP. The steady state depends on the parameters $\delta$ and $\gamma$. In the context of the model, the parameter $\delta$ can be interpreted as the quality of the existing infrastructure. Therefore, if the country's level of GDP has reached the level determined by the steady state, the only way to accelerate economic growth (taking into account the dependency on road infrastructure only) is to improve the quality of road infrastructure or, other words, to reduce $\delta .{ }^{9}$ This will be investigated in more detail in Section 4 .

\section{Data \& Calibration}

\subsection{Calibration Methods}

In this section we propose an approach to the calibration of the model if we are given statistical data relating a certain country. The model includes the following functions to be identify: $g(z), f(z), c(z), r(u)$. We assume that the parameters $\delta, \gamma, \mu$ and $\rho$ have been specified. Moreover, we have chosen forms for the functions $g(z), f(z), c(z)$ and $r(u)$ (see (9), (10), (11), (12)). So we need to identify the parameters $\alpha, \beta, A, B, D, E, L, \theta$.

\footnotetext{
${ }^{9}$ However, this result has to be seen with caution, since we should not forget that $u$ also depends on $\delta$ and so to find the "optimal" $\delta$ is not as straightforward as it seems because also the dependence of costs on the same would need to be considered in detail.
} 


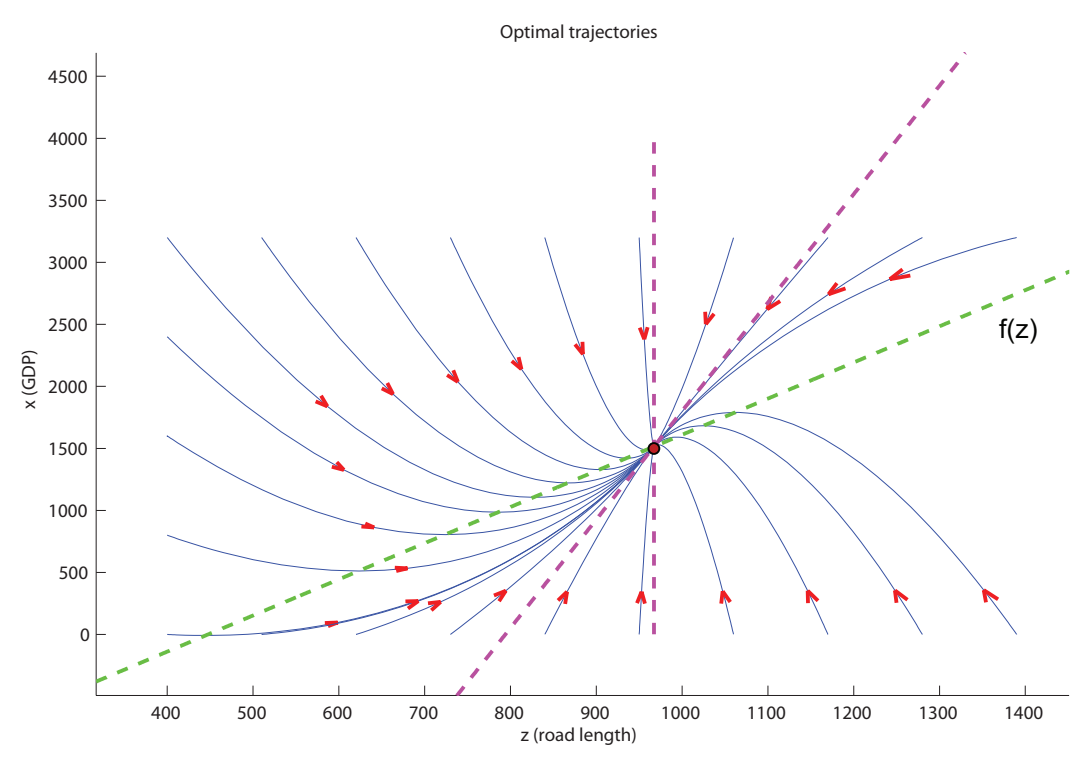

Figure 7: Optimal trajectories, asymptotic lines (magenta color) and steady state (red circle).

Let us assume that data are arranged as follows. All data are specified on a time grid that covers the time period $\left[t_{0}, T\right]$

$$
G_{T}=\left\{t_{0}, t_{1}, \ldots, t_{N_{T}}\right\}, \quad t_{N_{T}}=T .
$$

Assume that for every time moment from the time grid $G_{T}$ we have the following data (for a certain country):

$x_{i}-$ GDP value at time $t_{i}, i=0, \ldots, T_{N}$;

$z_{i}-$ capacity of road infrastructure at time $t_{i}, i=0, \ldots, T_{N}$

$c_{i}-$ maintenance cost at time $t_{i}, i=0, \ldots, T_{N}-1$;

$r_{i} \quad$ - building cost at time $t_{i}, i=0, \ldots, T_{N}-1$.

We split all parameters to be identify into three group. The first group is the parameters $\alpha$ and $\beta$ relating to the function $g(z)$. As the function $g(z)$ has been chosen linear (based on statistical data for various countries), linear regression can be used to calibrate $\alpha$ and $\beta$. The second group is the parameters $A$ and $B$ relating to the threshold function $A$ and $B$. It is not possible to calibrate the threshold line using statistical data for a single country. Therefore, we make the assumption that developed countries develop in the optimal way (in the sense of the described model). From this assumption follows that the optimal asymptote constructed for a given country has to coincide with the function $g(z)$ calibrated for the same country. That gives us equations to find $A$ and $B$ (see section (2.4)).

The third group is the parameters $D, E, L, \theta$ to be identified at the same time. When calibrating these parameters we take into account that the trajectory $z(t)$ has to satisfy the equation (1), consequently, we need to identify the control function $u(t)$ producing a given trajectory $\left\{z\left(t_{i}\right)\right\}_{i=0, \ldots, T_{N}}$ as well. The approach to calibrating these parameters is based on the the least-squares method. Let us introduce new variables $u_{i}$ that corresponds to the control $u\left(t_{i}\right), i=0, \ldots, N_{T}-1$, at time moment $t_{i}$. The equation (1) imposes the following constraints

$$
z_{i+1}-z_{i}=\left(u_{i}-\delta z_{i}\right)\left(t_{i+1}-t_{i}\right), \quad i=0, \ldots, N_{T}-1 .
$$


We need to minimize the function

$$
\sum_{i=0}^{N_{T}}\left[w_{c}\left(c_{i}-c\left(z_{i}\right)\right)^{2}\right]+\sum_{i=0}^{N_{T}-1}\left[w_{r}\left(r_{i}-r\left(u_{i}\right)\right)^{2}\right]
$$

under the constraints (24) by choosing $\left\{u_{i}\right\}_{i=0, \ldots, N_{T}-1}, D, E, L, \theta$. The coefficients $w_{c}, w_{r}$ should be chosen such that all items in the function (25) have the same scale.

Note that the constraints (24) enables to compute the variables $u_{i}$ directly

$$
u_{i}=\frac{z_{i+1}-z_{i}}{t_{i+1}-t_{i}}+\delta z_{i}, \quad i=0, \ldots, N_{T}-1 .
$$

Therefore, the minimization of the function (25) can be carried out for the first and second items independently. Taking into account that the function $c(z)$ is linear, we can apply linear regression to identify $D$ and $E$. The last step is to calibrate $r(u)$. Having plotted statistical data for the function $r(u)$, we obtain rather a grouped set of points located on a relatively small part of the plane $(u, r)$ than a curve. We assumed that the function $r(u)$ has the form

$$
r(u)=L\left(e^{\theta u}-1\right) .
$$

Therefore, we choose such values for the coefficients $L$ and $\theta$ that the exponential curve passes trough the set of points. In this case we get something like the extrapolation since the exponential curve satisfies the condition $r(0)=0$ (that must be imposed) and more or less approximates the group of points with a curve. To implement this approach, we can indicate (at least manually) a point located inside of the group of points and a slope at this point so that the exponential curves passes through the point and has specified slope.

Let $(\bar{u}, \bar{r})$ be a point which the curve has to pass through and $k$ be a curve slope in this point. We get the following equations to find $L$ and $\theta$ :

$$
\left\{\begin{array}{l}
L\left(e^{\theta \bar{u}}-1\right)=\bar{r} \\
L \theta e^{\theta \bar{u}}=k
\end{array}\right.
$$

Solving these equations, we get the nonlinear equation to find $\theta$ :

$$
1-\frac{\theta \bar{r}}{k}=\frac{1}{e^{\theta \bar{r}}}
$$

and equality to find $L$ :

$$
L=\frac{k}{\theta e^{\theta \bar{u}}}
$$

\subsection{Calibration Results}

Based on the data provided by Canning $(1998,1999)$ that we have been using above to motivate the functional forms of the relationships in the optimal control problem at hand, we have chosen to focus on two case studies: the two countries are Finland and France. We have chosen France as an example of one of the more mature economies with a relatively high income featuring in the upper right region in Figures 2 to 4 . Finland, on the other hand, is one the countries in the lower left corner of Figures 2 to 4 . If this is a matter of scale or whether it implies that these countries are farther removed from their steady state remains to be seen. The data for GDP are taken from the UNECE Statistical Division Database, compiled from national and international official sources such as EUROSTAT, and the OECD. 
Road length is from the UNECE Transport Division Database, which we have chosen instead of Canning's $(1998,1999)$ data because there is a larger overlap in time with the other series and our goal was to maximize the number of data points, since empirical applications are already subject to many points of criticism, so at least the data set should be as complete as possible. Investment and maintenance cost series are from the International Transport Forum, issued in May 2008.

Starting with GDP as a function of road infrastructure, we perform a linear regression of GDP and road length, where $A$ is the slope and $B$ the constant. The calibration results for both France and Finland show that this provides a very good fit compared to the actual data, judging from the high values we find for $R^{2}$.

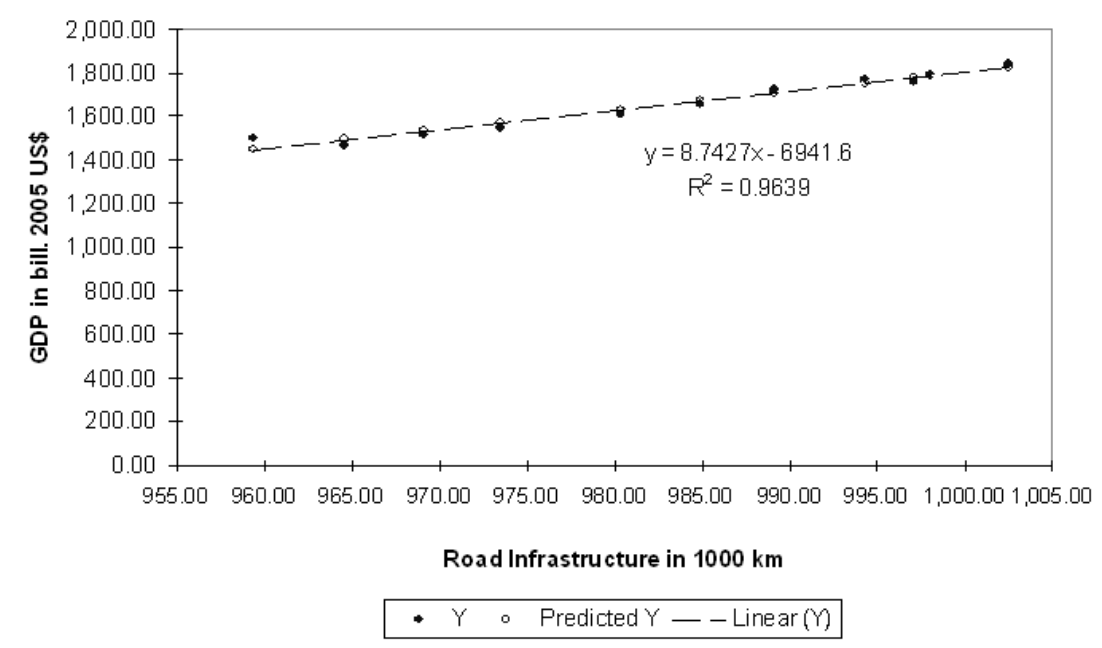

Figure 8: GDP as a function of road length, linear fit for France

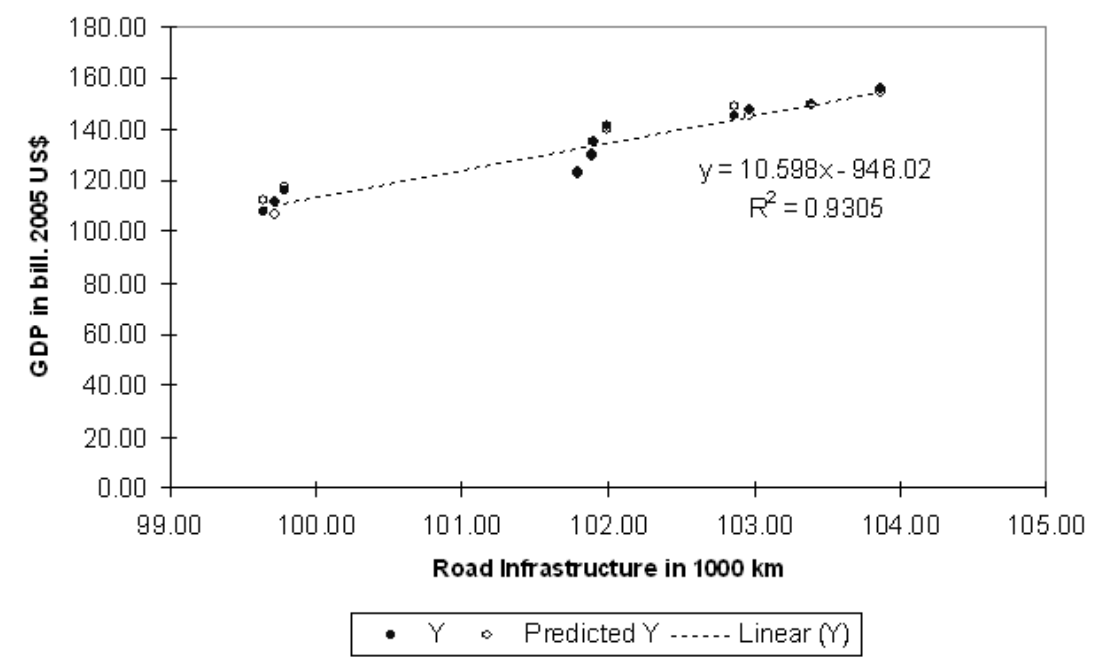

Figure 9: GDP as a function of road length, linear fit for Finland

The depreciation rates used in the calibration of building cost as a function of the growth in road length is 10 and $20 \%$ respectively for France and Finland. In order to smooth the series for the latter variable, we take the average of the difference in $u$ over 
the current and the coming year, where $u$ is computed as the difference between $z$ in the two years plus depreciation rate times current infrastructure. The exponential fit is then obtained by the method proposed in the previous section, i.e. we (manually) indicate a point located inside the group of points in the $(r, u)$-plane and a slope at this point so that the exponential curves passes through the point and has a specified slope. The results of this are displayed in Table 2 .

\begin{tabular}{l||r|r}
\hline Country & France & Finland \\
\hline specified point $(r, u)$ & $(12,100)$ & $(0.6,21)$ \\
\hline specified slope & 1.00 & 0.10 \\
\hline$\theta$ & 0.0833 & 0.1610 \\
$L$ & 0.0029 & 0.0211 \\
Fit & $21.79 \%$ & $10.81 \%$ \\
\hline \hline
\end{tabular}

Table 2: Building cost as a function of the growth in infrastructure capacity (The "Fit" is computed as the correspondence between the output (i.e. the predicted values) and the actual, observed data).

Even though the fit is far from perfect, we think that given the relative shortness of our time series and the justifications on the basis of the data set by Canning $(1998,1999)$ allow us to make use of the coefficients thus obtained, especially against the background that our results are not supposed to be numerically indicative of real developments, but rather illustrative of the new approach and the usefulness of applying optimum control theory to the problem of developing road infrastructure in a context of economic growth.

Finally, the relationship between maintenance cost and the existing road infrastructure stock is calibrated through linear regression again. Figure 10 above shows that this provides a very good fit in terms of $R^{2}$ for the case of France, while Finland has an $R^{2}$ of less than $2 \%$. Still, the linear fit seems the closest we can get to the behavior of the actual data. It is of course admissible to specify a different function for $c(z)$ for Finland, but this would require the re-computation of the analytical solution, which would not add to the illustrative character of this exposition and is thus beyond the scope of this paper.

\section{Optimal Control Results: Country Case Studies}

In Section 2 we have derived the analytical solutions for the long-run behavior of the economy and its convergence to a steady state, denoting the maximum attainable GDP with the required stock of road infrastructure, which is - inter alia - determined by the rate of physical decay or the quality of the roads and the speed, at which the economy adjusts, i.e. moves along its trajectory. While this might have seemed rather technical to the reader, we want to also emphasize the usefulness of the type of approach we have taken for real world problems and the associated policy agendas. In this section we therefore derive some results for the cases of France and Finland with the help of the data and calibration presented in the previous section.

Figures 12 and 13 display the phase diagrams for France and Finland respectively. The light dotted line (green in color-version) is the threshold curve. The darker, dashed line (pink in color-version) is the asymptote. The arrows of motion on the trajectories point to the steady state. The transparent dots correspond to the real data. For France, the results show that the country is currently below its long-run steady state and the same is true for Finland in Figure 13. 


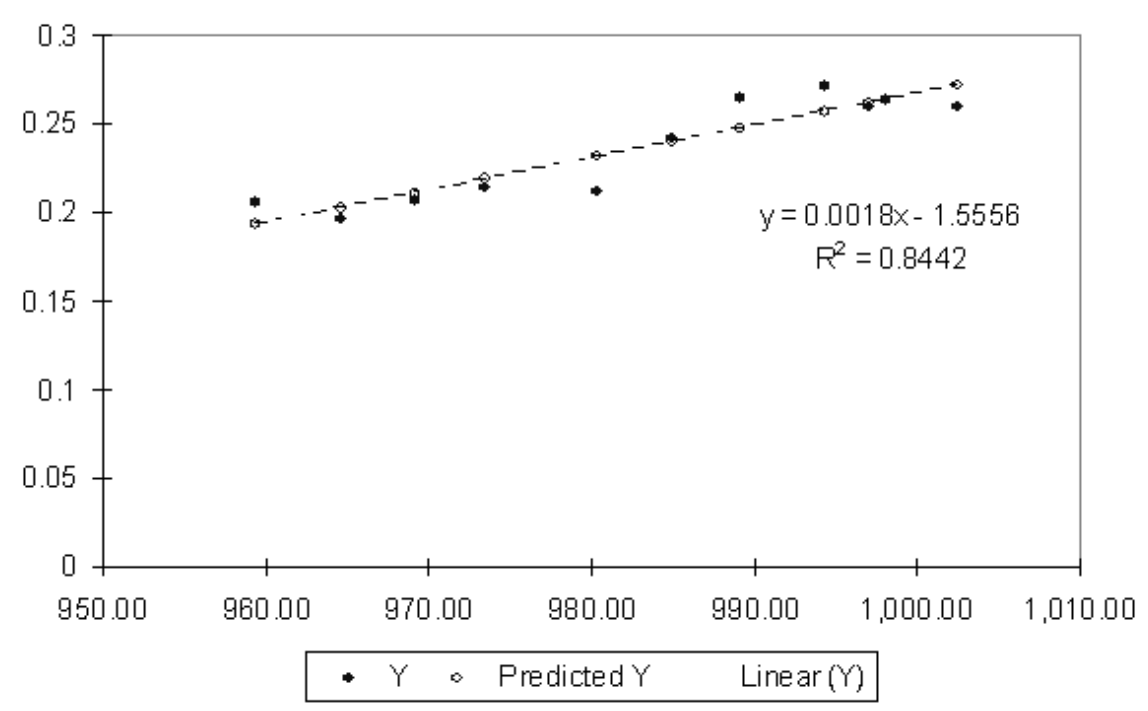

Figure 10: Maintenance cost as a function of road length, linear fit for France

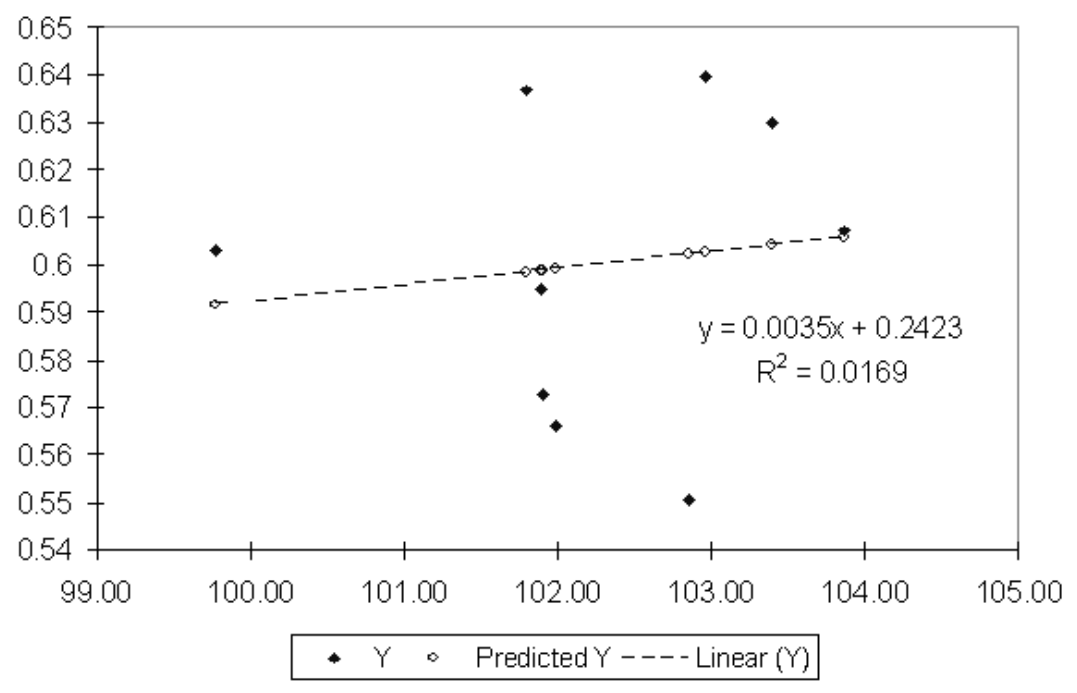

Figure 11: Maintenance cost as a function of road length, linear fit for Finland

Figure 14 demonstrates the sensitivity of the results with respect to the parameter $\delta$. If we interpret $\delta$ as an indicator of quality, which means that a lower value implies better quality, then the long-run steady state will indeed shift (remember our discussion at the end of Section 2) upwards and to the right and the economy will move along the asymptote to a higher long-run GDP level supported by a larger stock of higher-quality infrastructure.

\subsection{Infrastructure Quality \& Steady State GDP}

As mentioned in Section 2, it has been suggested that there is a relationship between the quality of existing infrastructure and steady state economic output. In the previous section it has been indicated that this relationship is positive (see Fig. 14). Analytically, it is difficult to find the value for $\delta$, which is "optimal" in the sense that it supports the 


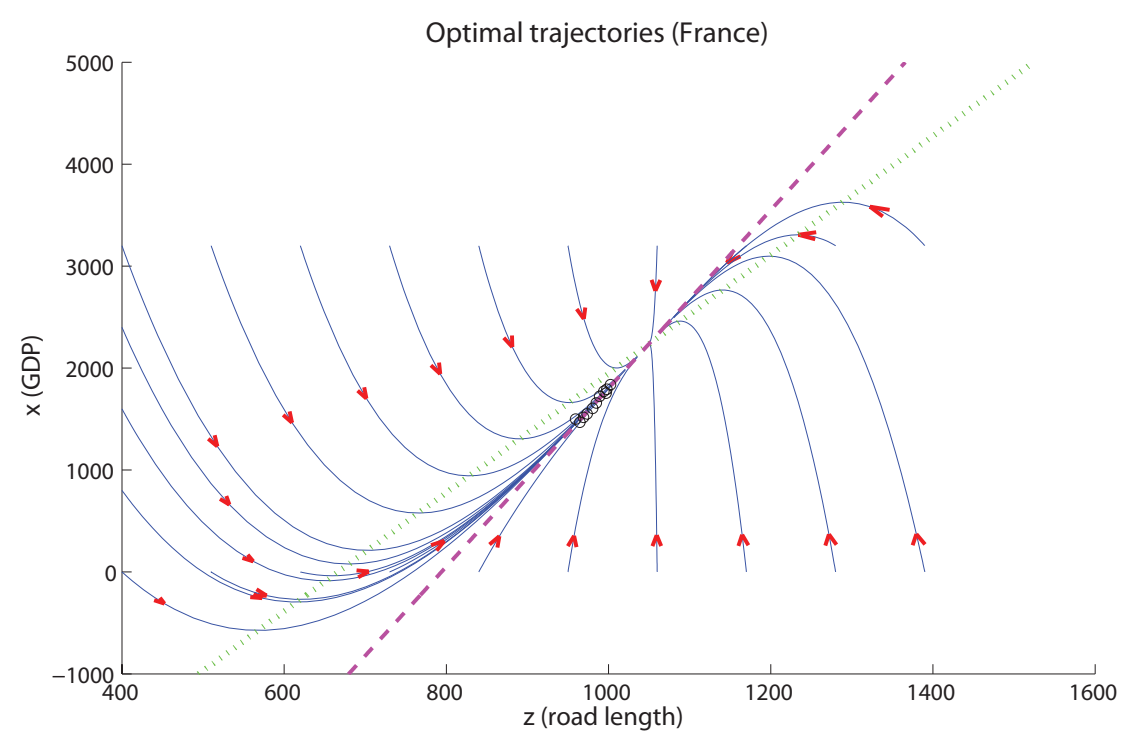

Figure 12: Phase diagram for France with trajectories

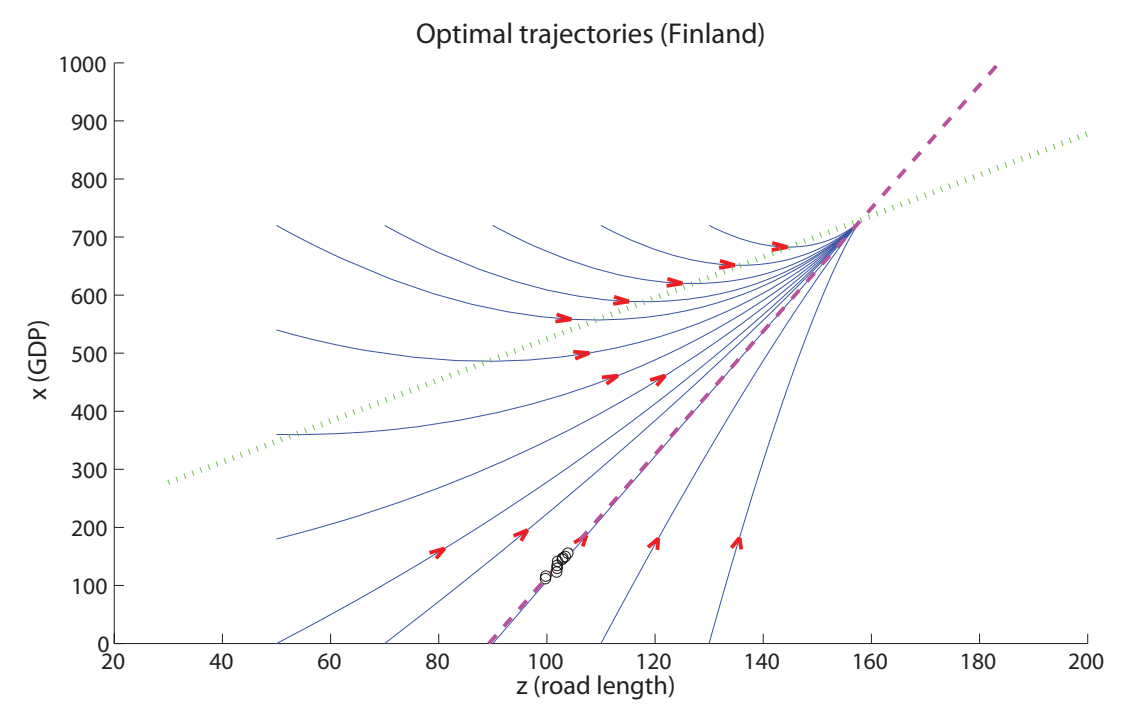

Figure 13: Phase diagram for Finland with trajectories

maximally attainable steady state GDP. The reason is that one would have to specify the exact dependence of costs on $\delta$, which has not been done here. Empirically - not knowing the precise value of $\delta$ - we can use the available data to calibrate the model for a given $\delta$ and find the optimal solution corresponding to that value. Plotting these optimal solutions for increasing values of $\delta$, we can then draw some conclusions about the relationship between infrastructure quality and steady state economic output.

Figures 15 and 16 show that for decreasing given $\delta$-representing increasing infrastructure quality according to our interpretation - a more than proportionately higher steady state GDP level can be attained in both France and Finland. Both graphs display similar properties.

The numerical results from this sensitivity exercise indicate that for a relatively small 


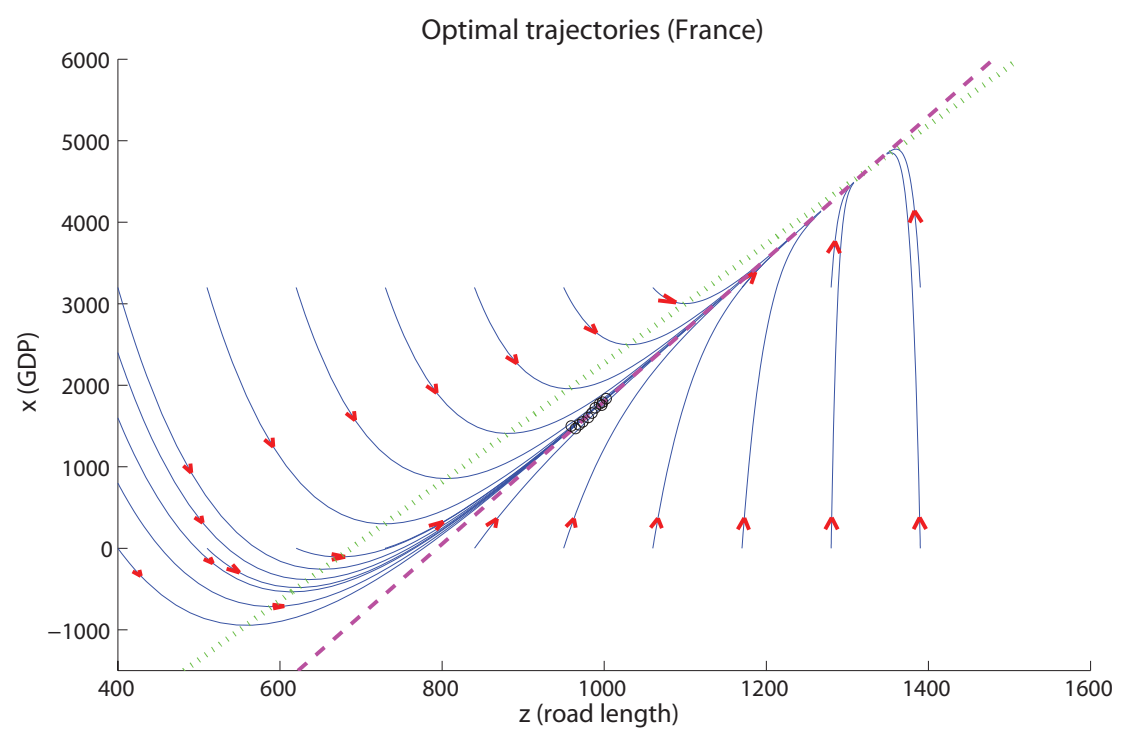

Figure 14: Phase diagram for France with better quality road infrastructure ( $\delta=5 \%)$

improvement in quality (a small decrease in $\delta$ ), a relatively large gain in terms of optimal GDP can be achieved. For relatively low levels of infrastructure quality (high $\delta$ ), the results should be looked at with scrutiny, since the model does not have a constraint with respect to the maximum impact of $\delta$ on steady state GDP and so the reader should not be misled to think that long run economic output could drop to zero or even negative levels if existing infrastructure deteriorates at a relatively fast pace.

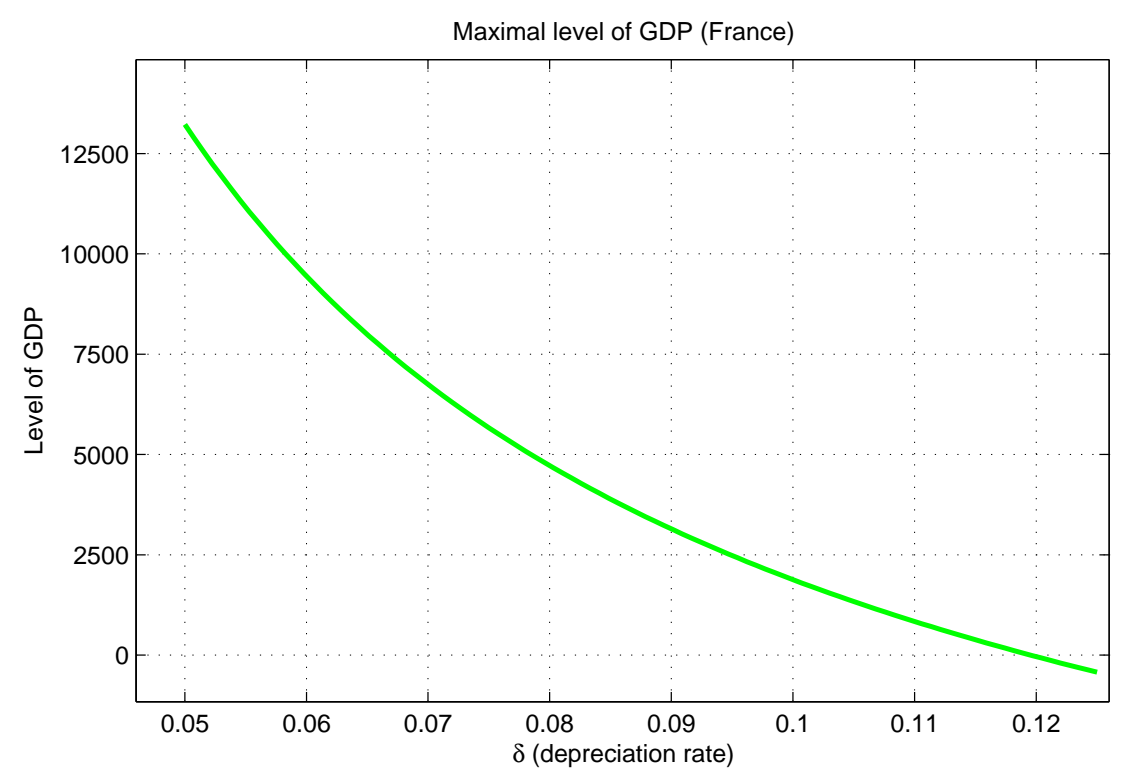

Figure 15: Steady state GDP for France against decreasing infrastructure quality (modeled as increasing $\delta$ ) 


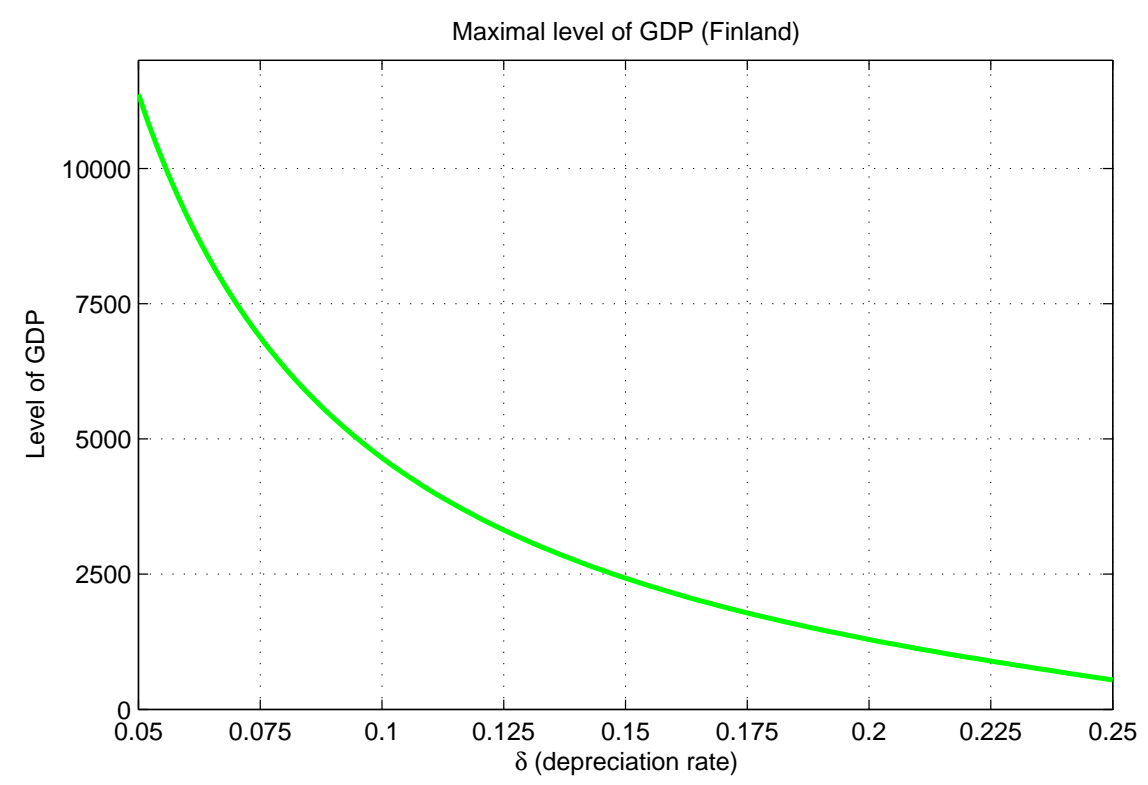

Figure 16: Steady state GDP for Finland against decreasing infrastructure quality (modeled as increasing $\delta$ )

\subsection{The Speed of Adjustment \& Steady State GDP}

Another parameter, which merits special attention in our model is $\gamma$. Looking back at Section 2.1 and, in particular, Equation 4, we remember that $\gamma$ is the coefficient of the speed of adjustment. It therefore represents the rate at which GDP approaches its longrun, optimal level when the economy is on one of the trajectories traced out in the previous sections. In this section we will test the relationship between different levels of $\gamma$ and steady state GDP for the calibrated cases of Finland and France. This sensitivity analysis will reveal how the ability of a country to adapt to its steady state influences the level that this steady state will have.

Remember from Figure 7 in Section 2 that there are two asymptotic lines in the model and that the trajectories converge along the inclined asymptotic line in the case of $\gamma>\delta$ and along the vertical asymptotic line in the case of $\gamma<\delta$ until the steady state is reached. In this section we focus on the first case, since in the other case the economy would adjust more slowly than its infrastructure deteriorates and without adding more constraints this could easily lead to negative growth and a contraction of the long-run economic output below zero.

With this caveat in mind, let us turn to Figure 17 and 18 displaying the results of the exercise for France and Finland respectively. In both cases it can be observed that the more quickly GDP approaches its long-run steady state level, the response of this level is initially huge and levels off afterwards, i.e. there is a level of maximal GDP that cannot be surpassed, no matter how large $\gamma$ is. In other words, the sensitivity analysis shows a positive but diminishing effect of the speed of adjustment on steady state GDP. 


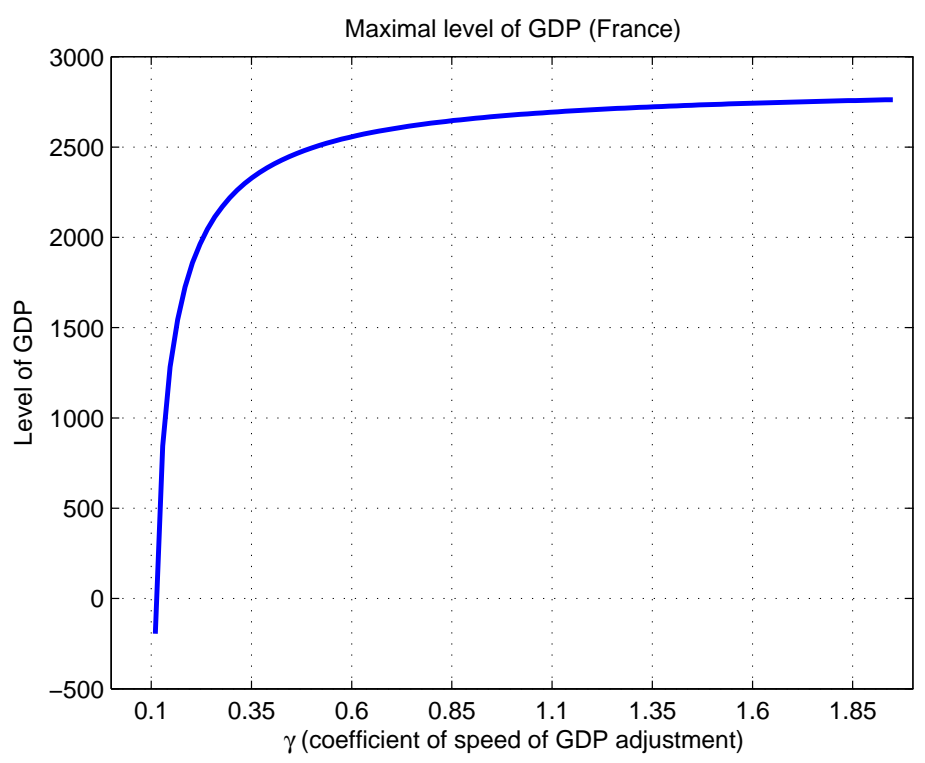

Figure 17: Steady state GDP for France against speed of adjustment (modeled as increasing $\gamma$ )

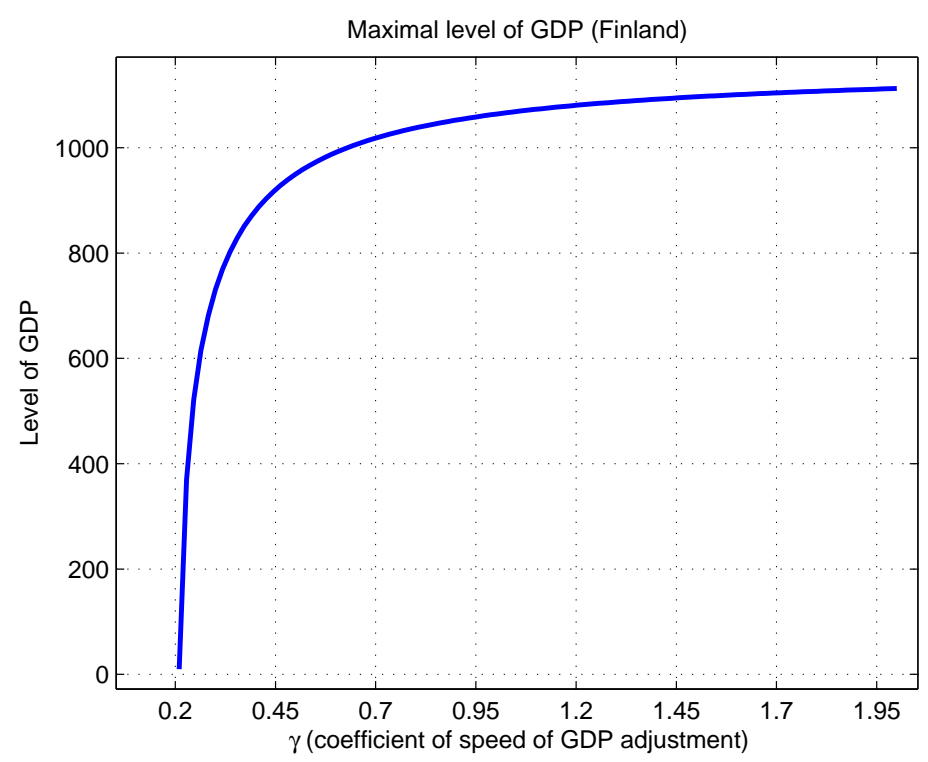

Figure 18: Steady state GDP for Finland against speed of adjustment (modeled as increasing $\gamma$ )

\subsection{The Share of Road Infrastructure in GDP \& Steady State GDP}

Remember that in Section 2.3, Table 1 shows a proxy of the portion of GDP, which is composed of road infrastructure, based on the amounts of energy used in road transport and the total energy consumed in the economy. Even though we opted for a rather cautious value of $\mu$ (5\%, which does not differ significantly from other studies' estimates (see Section 
2.3 ) in our case studies, Table 1 indicates that $\mu$ might be higher than that and, in addition, varies across countries. The values estimated for Finland grow from $12 \%$ to almost $15 \%$ in the period from 1980 to 1994; France starts out at $15 \%$ ending up at about $17 \%$.

The diversity of these figures raises the question how - in our case studies - the level of steady state GDP would be affected if we used a different $\mu$ in our framework with all other calibrated parameters unchanged. Therefore, we present below the corresponding sensitivity analysis for both France and Finland. Figures 19 and 20 display the same shape of relationship, where changing $\mu$ from a very small value to a slightly higher one has a very large impact on steady state economic output, but beyond $20 \%$ this effect levels off: while the graph continues to slope upwards, it is still slightly concave. Note that for a $\mu$ of $100 \%$ - even though impossible in a real world situation - France would be able to attain a long-run GDP level of US $\$ 5,000$ million; Finland's level would be about $\$ 1.700$ million.

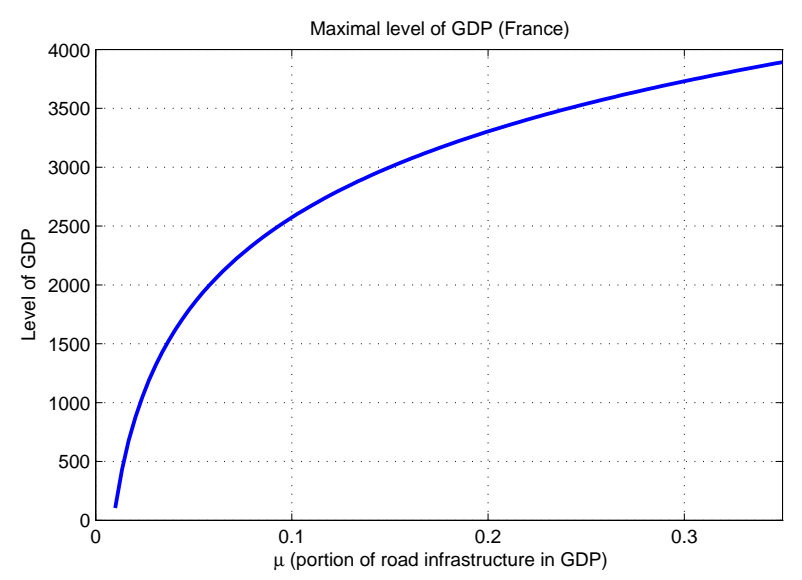

Figure 19: Steady state GDP for France against the share of road infrastructure in output (modeled as increasing $\mu$ )

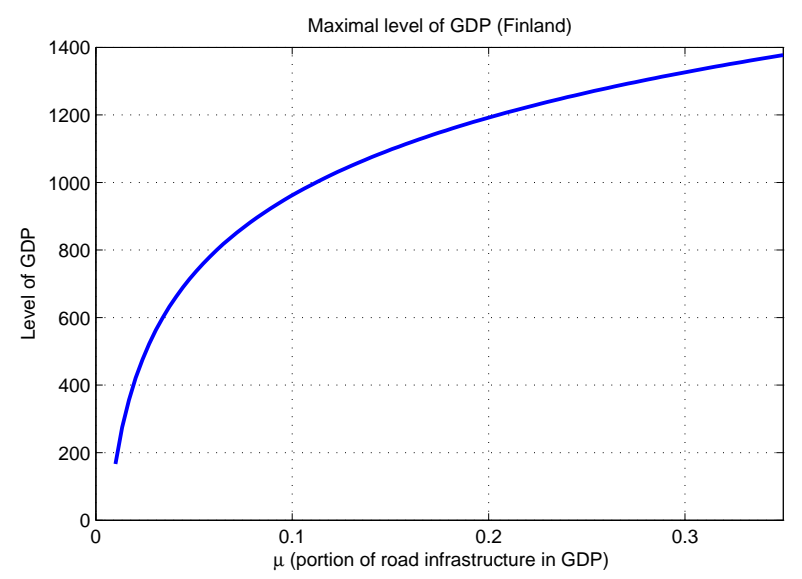

Figure 20: Steady state GDP for Finland against the share of road infrastructure in output (modeled as increasing $\mu$ ) 


\section{Summary and Conclusion}

In this paper we have applied optimal control theory to a co-evolutionary framework, where the co-evolving variables are economic output and road infrastructure. The control in our problem is additions to existing road capacity - in other words investment. Our goal was to show that useful insights can be derived from developing such an approach and when the underlying (cost) functions and constraints are adapted to a specific country or region, then policy makers can better inform their decisions about public investment into roads or about providing incentives for private road investment. We believe that a sound theoretical framework should be the foundation for further empirical work, the latter representing the major part of the literature, and therefore embark on demonstrating how this can be approached and that it can be calibrated and implemented if appropriate data are available.

We find an analytical solution for the infinite horizon problem, where the control turns out to be a constant. The steady state is shown to depend crucially on the rate of physical decay of roads, which we think can be interpreted as an index of quality, and the speed of adjustment, at which the economy moves along a trajectory. Another parameter, which merits special attention is the degree to which GDP is composed of road infrastructure as an input factor, the other factors being labor, resources, human capital, other types of physical capital, and so forth.

Testing the model for the data of two countries, France and Finland, illustrates the usefulness of such an approach to real world problems and possibly policy recommendation, where the model would have to be adapted to the peculiarities of each country or region to make precise statements. Sensitivity analyses with respect to the above-mentioned parameters show that larger quality of road infrastructure implies that a higher level of steady state GDP can be reached if the other calibrated relationships are unchanged. While this relationship is exponential, a larger speed of adjustment to steady state GDP and a larger portion of GDP being composed of road infrastructure are shown to have a concave relationship with long-run economic output. In other words, a marginal change at low levels of $\gamma$ and $\mu$ has a large impact on the output attainable in the long run, but this effect diminishes for larger values of these parameters.

We think that our study contributes to the existing literature by applying an existing modeling approach in combination with co-evolutionary features to a problem, which has previously mainly been the focus of empirical research and where there was much debate about causality issues and other problems when estimating the underlying relationships. Our model is admittedly simple, but this has been done on purpose, so as to illustrate the usefulness of our approach in a transparent and straightforward way. The empirical part (i.e. the country case studies) suffer from a lack of appropriate data to give robust estimates of the parameters used, but serve the goal of demonstrating that the framework can be adapted to real world analyses when the underlying relationships are adequately adapted to the situation and context.

Further research will be occupied with the extension of the current work in a spatial dimension. Furthermore, an effort will be made to collect a more comprehensive data set, as there is much scope for improvement on the empirical side. 


\section{References}

[1] Aschauer, D.A., Is Public Expenditure Productive? Journal of Monetary Economics, 23, 177-200, 1989.

[2] Aseev, S., and Kryazhimskiy, A., The Pontryagin Maximum Principle and Transversality Conditions for a Class of Optimal Control Problems with Infinite Time Horizons. IIASA Interim Report, RP-05-003, June 2005.

[3] B.P. plc., Statistical Review of World Energy 2008. B.P.

[4] Button, K., Infrastructure Investment, Endogenous Growth and Economic Convergence. The Annals of Regional Science 34(1), 145-162, 1998.

[5] Canning, D., A Database of World Stocks of Infrastructure, 1950-1995. World bank Economic Review, Oxford University press, 12(3), 529-547, 1998.

[6] Canning, D., Infrastructure's Contribution to Aggregate Output, Policy Research, Working Paper no. 2246, The World Bank, 1999.

[7] Dorfman, R., An Economic Interpretation of Optimal Control Theory. The American Economic Review 59(5), 817-831, 1969.

[8] European Conference of Ministers of Transport (ECMT). Statistical Trends in Transport 1965-1994. Cedex, France, 1998.

[9] Fedderke, J.W., Perkins, P., and Luiz, J.M., Infrastructural Investment in Long-run Economic Growth: South Africa 1875-2001. World Development 34(6), 1037-1059, 2006.

[10] French Road Federation Facts \& Figures. October 2006.

[11] Gramlich, E.M., Infrastructure Investment: A Review Essay, Journal of Economic Literature 32(3), 1176-1196, 1994.

[12] Grübler, A., and Nakicenovic, N., Evolution of Transport Systems: Past and Future. IIASA RR-91-8, June 1991.

[13] Hirschman, A.O., The Strategy of Economic Development. Yale University Press, New Haven, CT, 1958.

[14] Lee, E.B., and Markus, L., Foundations of Optimal Control Theory, John Wiley \& Sons, New York, 1967.

[15] Liberini, F., Economic Growth and Infrastructure Gap in Latin America, Rivista di Politica Economica 96(11/12), 145-186, 2006.

[16] Madison A., The world economy: Historical Statistics. HS-1: Western Europe 15012001. OECD Development Centre, 2001.

[17] Pontryagin, L.S., Boltyanskii, V.G., Gamkrelidze, R.V., and Mishchenko, E.F., Mathematical Theory of Optimal Processes, Interscience Publishers, John Wiley \& Sons, New York, 1962.

[18] Weidlich, W., et al, An integrated model of transport and urban evolution, Springer, 1999. 


\section{A Appendix: Optimal Control Problem with Finite Time Horizon}

Here we consider an optimal control problems that is similar to the problem described in the paper but the time horizon is finite

$$
\begin{aligned}
\text { maximize } J & =\int_{0}^{T} e^{-\rho t}\left(\mu x(t)-D z(t)-E-L e^{\theta u(t)}+L\right) d t \\
\text { subject to } & \\
\dot{z}(t) & =u(t)-\delta z(t) \\
\dot{x}(t) & =\gamma(A z(t)+B-x(t)) \\
u(t) & \in[0, \bar{u}] \\
z(0) & =z_{0} \\
x(0) & =x_{0}, \\
t & \in[0, T]
\end{aligned}
$$

where $T$ is a terminal (final) time moment that is specified. Note that the terminal state of the dynamic system, $x(T)$, is not fixed.

Following the maximum principle, let us compose the Hamilton-Pontryagin function

$$
\mathcal{H}(z, u, t, \psi)=e^{-\rho t}\left(\mu x-D z-E-L e^{\theta u}+L\right)+\psi_{1}(u-\delta z)+\psi_{2} \gamma(A z+B-x)
$$

and the adjoint equation

$$
\begin{cases}\dot{\psi}_{1}=-\frac{\partial \mathcal{H}}{\partial z}=\delta \psi_{1}-\gamma A \psi_{2}+D e^{-\rho t}, & \psi_{1}(T)=0, \\ \dot{\psi}_{2}=-\frac{\partial \mathcal{H}}{\partial x}=\gamma \psi_{2}-\mu e^{-\rho t}, & \psi_{2}(T)=0 .\end{cases}
$$

where $\psi_{1}(T)=0$ and $\psi_{2}(T)=0$ are the transversality condition for the case where the terminal state $x(T)$ is not fixed. Solving the adjoint equation we get

$$
\begin{aligned}
\psi_{1}(t)= & \frac{D \delta \rho+D \gamma \rho+\gamma A \mu \delta+D \gamma^{2}-D \gamma \delta-\gamma^{2} A \mu}{(\gamma+\rho)(\delta+\rho)(\delta-\gamma)} e^{-\rho t} \\
& +\frac{\rho \gamma A \mu-D \gamma \rho+D \delta \rho+\gamma^{2} A \mu+D \gamma \delta-D \gamma^{2}}{(\gamma+\rho)(\delta+\rho)(\delta-\gamma)} e^{\delta t-\rho T-\delta T} \\
& +\frac{-\rho \gamma A \mu-\gamma A \mu \delta}{(\gamma+\rho)(\delta+\rho)(\delta-\gamma)} e^{\gamma t-\rho T-\gamma T}, \\
\psi_{2}(t)= & \frac{\mu}{\gamma+\rho} e^{-\rho t}-\frac{\mu}{\gamma+\rho} e^{\gamma t-\rho T-\gamma T} .
\end{aligned}
$$

The extremal control satisfies the following maximum condition

$$
u(t, z, x, \psi)=\underset{u \in[0, \bar{u}]}{\arg \max } \mathcal{H}(t, z, x, u, \psi)=\underset{u \in[0, \bar{u}]}{\arg \max }\left\{\psi_{1} u-L e^{-\rho t} e^{\theta u}\right\} .
$$

Let us note, that the function

$$
M(u)=\psi_{1} u-L e^{-\rho t} e^{\theta u}
$$

is a concave function as $L>0$. Therefore,

$$
\underset{u \in[0, \bar{u}]}{\arg \max } M(u)= \begin{cases}0, & \hat{u}(t) \leq 0, \\ \hat{u}(t), & \hat{u}(t) \in(0, \bar{u}), \\ \bar{u}, & \hat{u}(t) \geq \bar{u},\end{cases}
$$


where $\hat{u}$ is a solution of the equation

$$
\frac{\partial M(u)}{\partial u}=0
$$

We get

$$
\hat{u}(t)=\frac{1}{\theta} \ln \left(\frac{e^{\rho t} \psi_{1}(t)}{L \theta}\right) .
$$

Finally, we get the optimal control

$$
u^{*}(t)= \begin{cases}0, & \hat{u}(t) \leq 0 \\ \hat{u}(t), & \hat{u}(t) \in(0, \bar{u}) \\ \bar{u}, & \hat{u}(t) \geq \bar{u}\end{cases}
$$

where

$$
\begin{gathered}
\hat{u}(t)=\frac{1}{\theta} \ln \left(\eta-\xi e^{(\delta+\rho)(t-T)}-\zeta e^{(\gamma+\rho)(t-T)}\right), \\
\eta=\frac{\gamma A \mu-D \gamma-D \rho}{L \theta(\gamma+\rho)(\delta+\rho)} \\
\xi=\frac{D \gamma-D \delta-\gamma A \mu}{L \theta(\delta+\rho)(\delta-\gamma)} \\
\zeta=\frac{\gamma A \mu}{L \theta(\gamma+\rho)(\delta-\gamma)}
\end{gathered}
$$

Let us assume now that the final instant of time, $\mathrm{T}$, goes to infinity. Then the items

$$
\xi e^{(\delta+\rho)(t-T)}, \quad \zeta e^{(\gamma+\rho)(t-T)}
$$

are close to zero. Therefore,

$$
\hat{u}(t) \approx \tilde{u}=\frac{\ln (\eta)}{\theta} .
$$

So we get that the optimal control is a constant function and has the form

$$
u^{*}(t)= \begin{cases}0, & \tilde{u} \leq 0 \\ \tilde{u}, & \tilde{u} \in(0, \bar{u}) \\ \bar{u}, & \tilde{u} \geq \bar{u}\end{cases}
$$

where

$$
\tilde{u}=\frac{1}{\theta} \ln \left(\frac{\gamma A \mu-D \gamma-D \rho}{L \theta(\gamma+\rho)(\delta+\rho)}\right)
$$

\section{B Appendix: Some Additional Data}

In order to see some qualitatively distinct features of distinct transport infrastructures, it is illustrative to see the plot of total railroad vs. GDP, where data from the ECMT (1998) are shown. In most countries the curves show a declining trend, although GDP is growing. Thus, the choice of railroad infrastructure would not match our model. There is an important reason for that. According to Nakicenovic and Grübler (1991), all transportation technologies undergo a process that start with a growing trend, eventually reach 


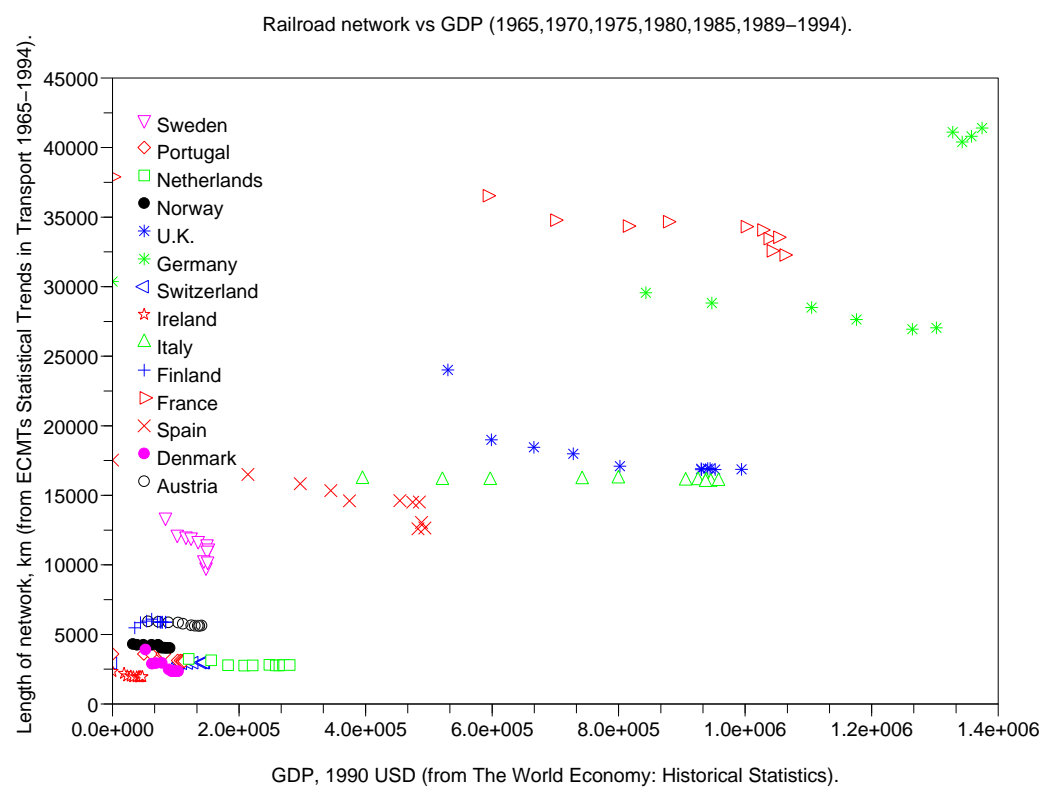

Figure B.1: Length of railroad network vs GDP level.

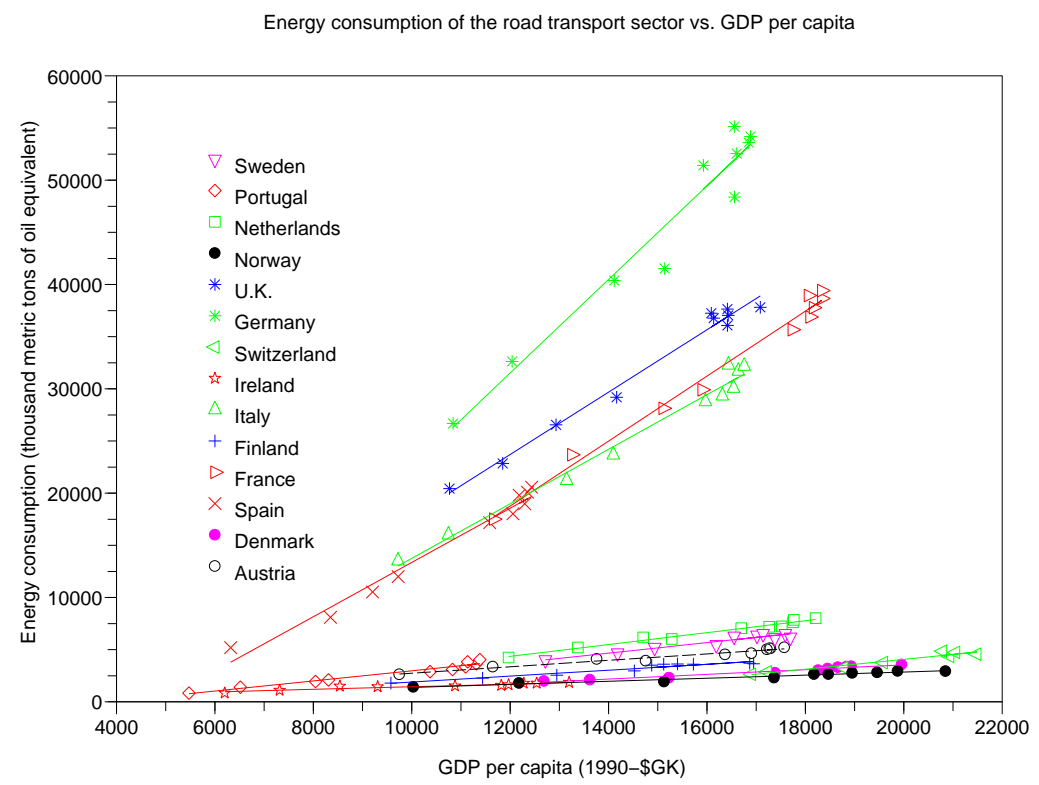

Figure B.2: Energy consumption in road transport vs. GDP per capita.

a saturation level and finally show a decline, to be then replaced by the next generation of transportation technology.

Energy used in the road transport sector is an indicator of economic activity, as it was already shown in Figure 4, where a clear linear relationship between the energy consumption in the road transport sector and GDP can be observed. As a means of characterizing distinctive particular features of the relation between the countries in connection with economic activity in the road transport sector, Figures B.2 and B.3 show relations between 
the energy consumed in the road transport sector and different measures of concentration of GDP.

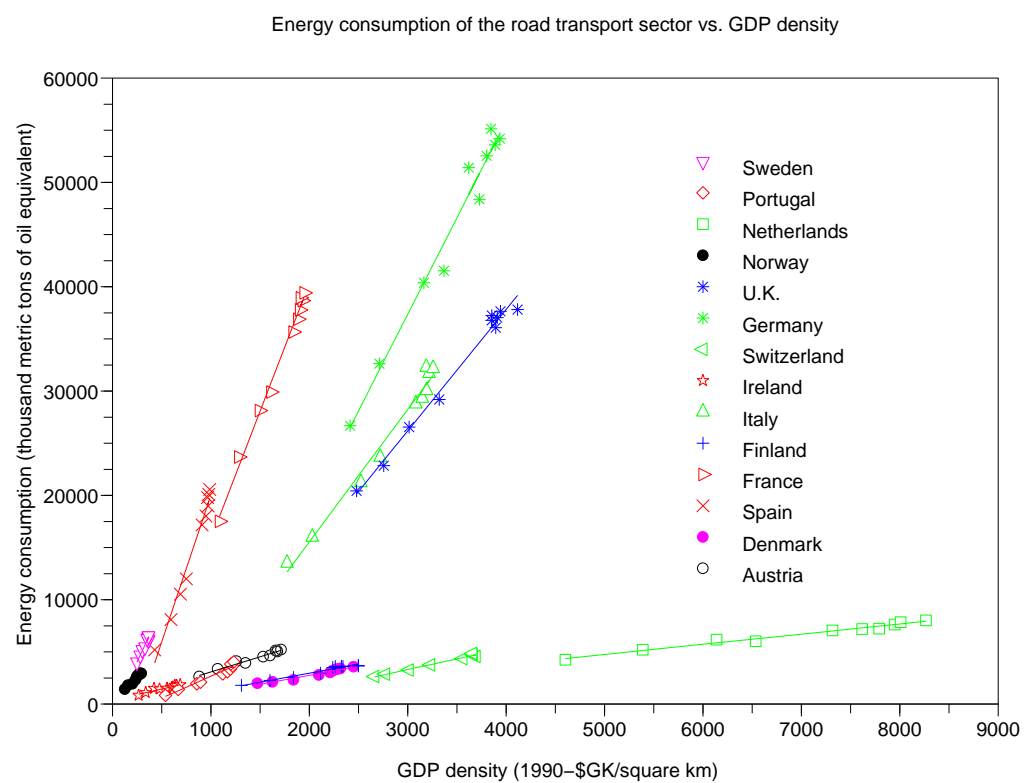

Figure B.3: Energy consumption in road transport vs. GDP density.

\begin{tabular}{llc}
\hline & & \\
& GDP per capita & GDP density \\
\hline Germany & 4.484 & \\
France & 3.113 & 18.517 \\
U.K. & 2.992 & 11.637 \\
Italy & 2.614 & 12.701 \\
Spain & 2.596 & 28.210 \\
Austria & 0.308 & 2.925 \\
Netherlands & 0.569 & 0.975 \\
Sweden & 0.502 & 20.286 \\
Portugal & 0.488 & 4.201 \\
Switzerland & 0.472 & 2.049 \\
Finland & 0.287 & 1.720 \\
Denmark & 0.227 & 1.733 \\
Norway & 0.148 & 9.617 \\
Ireland & 0.125 & 2.126 \\
\hline
\end{tabular}

Table B.1: Slopes of the plots road energy, Figures B.2 and B.3.

A first notable feature is that most plots show linear dependence. This allows us to characterize the intensity of energy usage in the road sector that is necessary to for an increase of the given GDP concentration index in terms of the slope. In the case of GDP per capita ( $2^{\text {nd }}$ column), the greatest values correspond to Germany, France, United Kingdom, Italy and Spain, and the smallest to Ireland. On the other hand, for GDP 
density ( $3^{\text {rd }}$ column), the greatest values show distinct ordering, although the countries with the greatest values for the $2^{\text {nd }}$ column are still in the group of the countries with the greatest values, but this time Sweden and Norway have high coefficients. 